\title{
AN APPLICATION OF SPHERICAL GEOMETRY TO HYPERKÄHLER SLICES
}

\author{
PETER CROOKS AND MAARTEN VAN PRUIJSSEN
}

\begin{abstract}
This work is concerned with Bielawski's hyperkähler slices in the cotangent bundles of homogeneous affine varieties. One can associate such a slice to the data of a complex semisimple Lie group $G$, a reductive subgroup $H \subseteq G$, and a Slodowy slice $S \subseteq \mathfrak{g}:=\operatorname{Lie}(G)$, defining it to be the hyperkähler quotient of $T^{*}(G / H) \times(G \times S)$ by a maximal compact subgroup of $G$. This hyperkähler slice is empty in some of the most elementary cases (e.g. when $S$ is regular and $(G, H)=\left(\mathrm{SL}_{n+1}, \mathrm{GL}_{n}\right)$, $n \geq 3$ ), prompting us to seek necessary and sufficient conditions for non-emptiness.

We give a spherical-geometric characterization of the non-empty hyperkähler slices that arise when $S=S_{\text {reg }}$ is a regular Slodowy slice, proving that non-emptiness is equivalent to the so-called $\mathfrak{a}$-regularity of $(G, H)$. This a-regularity condition is formulated in several equivalent ways, one being a concrete condition on the rank and complexity of $G / H$. We also provide a classification of the a-regular pairs $(G, H)$ in which $H$ is a reductive spherical subgroup. Our arguments make essential use of Knop's results on moment map images and Losev's algorithm for computing Cartan spaces.
\end{abstract}

\section{Contents}

1. Introduction

1.1. Context

1.2. Description of results

1.3. Organization

Acknowledgements

2. Preliminaries

2.1. Symplectic varieties and quotients

2.2. Hyperkähler manifolds

3. The hyperkähler geometry of $T^{*}(G / H)$

3.1. The cotangent bundle of $G$

3.2. Kronheimer's hyperkähler structure on $T^{*} G$

3.3. The hyperkähler structure on $T^{*}(G / H)$

4. The hyperkähler slice construction

4.1. The slice as a symplectic variety

4.2. Bielawski's construction

4.3. The regular Slodowy slice

5. The spherical geometry of $G / H$

5.1. The image of the moment map.

5.2. a-regularity

5.3. The Cartan space of a homogeneous affine variety

References

2010 Mathematics Subject Classification. 20G20 (primary); 53C26, $14 \mathrm{M} 17$ (secondary).

Key words and phrases. hyperkähler quotient, Slodowy slice, spherical geometry. 


\section{INTRODUCTION}

1.1. Context. A smooth manifold is called hyperkähler if it comes equipped with three Kähler structures that determine the same Riemannian metric, and whose underlying complex structures satisfy certain quaternionic identities. Such manifolds are known to be holomorphic symplectic and Calabi-Yau, and they are ubiquitous in modern algebraic and symplectic geometry. Prominent examples include the cotangent bundles [22] and (co-)adjoint orbits 6, 19,23, 24] of complex semisimple Lie groups, moduli spaces of Higgs bundles over compact Riemann surfaces [14], and Nakajima quiver varieties [29, 30]. Many examples arise via the hyperkähler quotient construction [15], an analogue of symplectic reduction for a hyperkähler manifold endowed with a structure-preserving Lie group action and a hyperkähler moment map. However, one always has the preliminary problem of determining whether the given hyperkähler quotient is non-empty.

While the above-described emptiness problem is likely intractable in the generality described above, one might hope to solve it for particular classes of hyperkähler quotients. It is in this context that one might consider Bielawski's hyperkähler slices [4,5], which require fixing a compact, connected, semisimple Lie group $K$ with complexification $G:=K_{\mathbb{C}}$. Each $\mathfrak{s l}_{2}$-triple $\tau=(\xi, h, \eta)$ in $\mathfrak{g}:=\operatorname{Lie}(G)$ determines a Slodowy slice $S_{\tau}:=\xi+\operatorname{ker}\left(\operatorname{ad}_{\eta}\right) \subseteq \mathfrak{g}$, and hence also an affine variety $G \times S_{\tau}$. This variety is a hyperkähler manifold carrying a tri-Hamiltonian action of $K$, and its symplectic geometry is reasonably well-studied (see [1,4,8,9]). Now suppose that $K$ acts in a tri-Hamiltonian fashion on a hyperkähler manifold $M$, and that this action extends to a holomorphic, Hamiltonian $G$-action with respect to the holomorphic symplectic structure on $M$. The hyperkähler slice for $M$ and $\tau$ is then defined to be $\left(M \times\left(G \times S_{\tau}\right)\right) / / / K$, the hyperkähler quotient of $M \times\left(G \times S_{\tau}\right)$ by $K$. Several well-known hyperkähler manifolds are realizable as hyperkähler slices, as discussed in the introduction of [5].

In light of the preceding discussion, one might consider the following special case of the emptiness problem: classify those pairs $(M, \tau)$ for which the hyperkähler slice $\left(M \times\left(G \times S_{\tau}\right)\right) / / K$ is non-empty. An initial objection is that no particular assumptions have been made about $M$ and $\tau$, so that this problem likely remains too general to be tractable. We thus note that the best studied Slodowy slices are those associated to regular $\mathfrak{s l}_{2}$-triples $\tau$ (see [18]), i.e. those $\tau=(\xi, h, \eta)$ for which $\xi$ is a regular element of $\mathfrak{g}$. At the same time, some of the best understood hyperkähler manifolds take the form of $T^{*}(G / H)$ for $H \subseteq G$ a closed, reductive subgroup (see [10]). We therefore study the emptiness problem for hyperkähler slices when $\tau$ is regular and $M=T^{*}(G / H)$.

Having decided to study hyperkähler slices in $T^{*}(G / H)$, we are naturally led to examine the Hamiltonian geometry of $T^{*}(G / H)$. The works of Knop [16, 17] encode this Hamiltonian geometry in the spherical geometry of $G / H$, by which we mean the $B$-orbit structure of $G / H$ for a Borel subgroup $B \subseteq G$. Fix such a subgroup $B \subseteq G$ and a maximal torus $T \subseteq B$ having Lie algebra $\mathfrak{t} \subseteq \mathfrak{g}$. Knop uses the Cartan space $\mathfrak{a}_{G / H}^{*} \subseteq \mathfrak{t}^{*}$ to describe the (closure of the) moment map image of $T^{*}(G / H)$. This is complemented by Losev's work [25], which gives an algorithm for calculating the Cartan space of any given affine homogeneous $G$-variety [25]. It is thus reasonable to imagine that spherical-geometric ideas are relevant to our specific emptiness problem.

1.2. Description of results. Let all notation be as set in the previous subsection, and write $S_{\text {reg }}$ for the Slodowy slice determined by a regular $\mathfrak{s l}_{2}$-triple $\tau$ in $\mathfrak{g}$. Use the Killing form to identify $\mathfrak{g}^{*}$ with $\mathfrak{g}$, and let $\mu: T^{*}(G / H) \rightarrow \mathfrak{g}$ be the moment map of the Hamiltonian $G$-action on $T^{*}(G / H)$. We note the existence of a non-negative, $K$-invariant potential function for the first Kähler triple on $T^{*}(G / H)$ (Proposition 44), which by Bielawski's results [4] implies that $\left(T^{*}(G / H) \times\left(G \times S_{\mathrm{reg}}\right)\right) / / / K$ and $\mu^{-1}\left(S_{\text {reg }}\right)$ are canonically isomorphic as holomorphic symplectic manifolds. This isomorphism is subsequently used to prove that $\left(T^{*}(G / H) \times\left(G \times S_{\text {reg }}\right)\right) / / K \neq \emptyset$ if and only if $\mathfrak{h}^{\perp}$ contains a regular element of $\mathfrak{g}$ (Proposition 11), where $\mathfrak{h}^{\perp} \subseteq \mathfrak{g}$ denotes the annihilator of $\mathfrak{h}:=\operatorname{Lie}(H)$ under the Killing form. The emptiness problem for $\left(T^{*}(G / H) \times\left(G \times S_{\text {reg }}\right)\right) / / K$ thus reduces to classifying the 
pairs $(G, H)$ for which $\mathfrak{h}^{\perp}$ contains a regular element. This is the stage at which spherical geometry becomes relevant, as we explain below.

Inside of $G$, fix a maximal torus $T$ and a Borel subgroup $B$ satisfying $T \subseteq B$. These choices allow us to form the Cartan space of $G / H$, denoted $\mathfrak{a}_{G / H} \subseteq \mathfrak{t}:=\operatorname{Lie}(T)$. We refer to the pair $(G, H)$ as being $\mathfrak{a}$-regular if $\mathfrak{a}_{G / H}$ contains a regular element of $\mathfrak{g}$, and we use Knop's description of the moment map image $\mu\left(T^{*}(G / H)\right)$ to prove the following equivalences (see Proposition 15, Corollary 17, and Corollary [19):

$$
\begin{aligned}
(G, H) \text { is a-regular } & \Longleftrightarrow \mathfrak{h}^{\perp} \text { contains a regular element } \\
& \Longleftrightarrow Z_{G}\left(\mathfrak{a}_{G / H}\right)=T \\
& \Longleftrightarrow \text { the identity component of } H_{*} \text { is abelian } \\
& \Longleftrightarrow c_{G}(G / H)+\operatorname{rk}_{G}(G / H)+\operatorname{dim} H=\operatorname{dim} B,
\end{aligned}
$$

where $Z_{G}\left(\mathfrak{a}_{G / H}\right)$ is the subgroup consisting of all elements in $G$ that fix $\mathfrak{a}_{G / H}$ pointwise, $H_{*}$ is the generic stabilizer for the $H$-representation $\mathfrak{h}^{\perp}$ (see 5.2), $c_{G}(G / H)$ is the complexity of $G / H$, and $\operatorname{rk}_{G}(G / H)$ is the rank of $G / H$. The first equivalence further reduces our emptiness problem to one of classifying the a-regular pairs $(G, H)$, thereby connecting our work to Losev's results [25]. We then classify all such pairs $(G, H)$ (i.e. we solve the emptiness problem for $\left.\left(T^{*}(G / H) \times\left(G \times S_{\text {reg }}\right)\right) / / / K\right)$ in each of the following three cases:

- $G$ is semisimple and $H$ is a Levi subgroup of $G$ (5.5.1);

- $G$ is semisimple and $H$ is a symmetric subgroup of $G$ (5.5.2);

- $G$ is semisimple and $H$ is a reductive, spherical, non-symmetric subgroup of $G$ (5.5.3).

In each case, we reduce to the study of strictly indecomposable (see 5.3) pairs $(G, H)$. It is in the last two cases that we obtain the most explicit results, and where we provide tables of all $\mathfrak{a}$-regular pairs $(G, H)$ that are strictly indecomposable.

1.3. Organization. Section 2 establishes some of our conventions regarding symplectic and hyperkähler geometry. Section 3 then uses [10], 22], and [27] to develop the hyperkähler-geometric features of $T^{*}(G / H)$ needed for the subsequent discussion of hyperkähler slices. This leads to Section 4, which reviews Bielawski's hyperkähler slice construction and reduces the non-emptiness of $\left(T^{*}(G / H) \times\left(G \times S_{\text {reg }}\right)\right) / / / K$ to the condition that $\mathfrak{h}^{\perp}$ contain a regular element. Section 5 then forms the spherical-geometric part of our paper, where we prove the equivalences (1) and subsequently obtain our classification results.

Acknowledgements. The central themes of this paper were developed at the Hausdorff Research Institute for Mathematics (HIM), while both authors took part in the HIM-sponsored program Symplectic geometry and representation theory. We gratefully acknowledge the HIM for its hospitality and stimulating atmosphere. We also wish to recognize Steven Rayan and Markus Röser for enlightening conversations. The first author is supported by the Natural Sciences and Engineering Research Council of Canada [516638-2018].

\section{PRELIMINARIES}

2.1. Symplectic varieties and quotients. Let $(X, \omega)$ be a symplectic variety, which for us shall always mean that $X$ is a smooth affine algebraic variety over $\mathbb{C}$ equipped with an algebraic symplectic form $\omega \in \Omega^{2}(X)$. Suppose that $X$ is acted upon algebraically by a connected complex reductive algebraic group $G$ having Lie algebra $\mathfrak{g}$. We recall that this action is called Hamiltonian if it preserves $\omega$ and admits a moment map, i.e. a $G$-equivariant variety morphism $\mu: X \rightarrow \mathfrak{g}^{*}$ satisfying the following condition:

$$
d\left(\mu^{z}\right)=\iota_{z} \omega
$$


for all $z \in \mathfrak{g}$, where $\mu^{z}: X \rightarrow \mathbb{C}$ is defined by $\mu^{z}(x):=(\mu(x))(z), x \in X$, and $\tilde{z}$ is the fundamental vector field on $X$ associated to $z$. If the $G$-action is also free, then

$$
X / / G:=\mu^{-1}(0) / G:=\operatorname{Spec}_{\max }\left(\mathbb{C}\left[\mu^{-1}(0)\right]^{G}\right)
$$

is a smooth affine variety whose points are precisely the $G$-orbits in $\mu^{-1}(0)$. The quotient variety $X / / G$ then carries a symplectic form $\bar{\omega}$ that is characterized by the condition $\pi^{*}(\bar{\omega})=j^{*}(\omega)$, where $\pi: \mu^{-1}(0) \rightarrow X / / G$ is the quotient map and $j: \mu^{-1}(0) \rightarrow X$ is the inclusion. The symplectic variety $(X / / G, \bar{\omega})$ is called the symplectic quotient of $X$ by $G$.

2.2. Hyperkähler manifolds. Recall that a smooth manifold $M$ is called hyperkähler if it comes equipped with three (integrable) complex structures $I_{1}, I_{2}$, and $I_{3}$, three (real) symplectic forms $\omega_{1}$, $\omega_{2}$, and $\omega_{3}$, and a single Riemannian metric $b$, subject the following conditions:

- $\left(I_{\ell}, \omega_{\ell}, b\right)$ is a Kähler triple for each $\ell=1,2,3$, i.e. $\omega_{\ell}(\cdot, \cdot)=b\left(I_{\ell}(\cdot), \cdot\right)$;

- $I_{1}, I_{2}$, and $I_{3}$ satisfy the quaternionic identities $I_{1} I_{2}=I_{3}=-I_{2} I_{1}, I_{1} I_{3}=-I_{2}=-I_{3} I_{1}$, $I_{2} I_{3}=I_{1}=-I_{3} I_{2}$.

One may construct new examples from existing ones via the hyperkähler quotient construction, which we now recall. Let $K$ be a compact connected Lie group acting freely on a hyperkähler manifold $M$, and let $\mathfrak{k}$ be the Lie algebra of $K$. Assume that the $K$-action is tri-Hamiltonian, meaning that $K$ preserves each Kähler triple $\left(I_{\ell}, \omega_{\ell}, b\right)$ and acts in a Hamiltonian fashion with respect to each symplectic form $\omega_{\ell}$. One thus has a hyperkähler moment map, i.e. a map $\mu_{\mathrm{HK}}=\left(\mu_{1}, \mu_{2}, \mu_{3}\right): M \rightarrow$ $\mathfrak{k}^{*} \oplus \mathfrak{k}^{*} \oplus \mathfrak{k}^{*}$ with the property that $\mu_{\ell}: M \rightarrow \mathfrak{k}^{*}$ is a moment map for the $K$-action with respect to $\omega_{\ell}, \ell=1,2,3$. The smooth manifold

$$
M / / K:=\mu_{\mathrm{HK}}^{-1}(0) / K=\left(\mu_{1}^{-1}(0) \cap \mu_{2}^{-1}(0) \cap \mu_{3}^{-1}(0)\right) / K
$$

is then canonically hyperkähler (see [15, Theorem 3.2]), and it is called the hyperkähler quotient of $M$ by $K$. We shall let $\left(\bar{I}_{\ell}, \bar{\omega}_{\ell}, \bar{b}\right), \ell=1,2,3$, denote the three Kähler triples that constitute the hyperkähler structure on $M / / / K$. It will be advantageous to note that

$$
\pi^{*}\left(\bar{\omega}_{\ell}\right)=j^{*}\left(\omega_{\ell}\right), \quad \ell=1,2,3,
$$

where $\pi: \mu^{-1}(0) \rightarrow M / / K$ is the quotient map and $j: \mu^{-1}(0) \rightarrow M$ is the inclusion.

Let $M$ be a hyperkähler manifold and consider the complex symplectic 2-form $\omega_{\mathbb{C}}:=\omega_{2}+i \omega_{3}$. One can verify that $\omega_{\mathbb{C}}$ is holomorphic with respect to $I_{1}$, and we will refer to $\left(M, I_{1}, \omega_{\mathbb{C}}\right)$ as the underlying holomorphic symplectic manifold. This leads to the following definition, which will apply to many situations of interest in our paper.

Definition 1. Let $K$ be a compact connected Lie group with complexification $G:=K_{\mathbb{C}}$. We define a $(G, K)$-hyperkähler variety is a to be a hyperkähler manifold $M$ satisfying the following conditions:

(i) the underlying holomorphic symplectic manifold is a symplectic variety (as defined in 2.1), and this variety is equipped with a Hamiltonian action of $G$;

(ii) the $G$-action restricts to a tri-Hamiltonian action of $K$ on $M$.

Consider the hyperkähler moment map $\mu_{\mathrm{HK}}=\left(\mu_{1}, \mu_{2}, \mu_{3}\right): M \rightarrow \mathfrak{k}^{*} \oplus \mathfrak{k}^{*} \oplus \mathfrak{k}^{*}$ on a $(G, K)$ hyperkähler variety $M$. Define the complex moment map by

$$
\mu_{\mathbb{C}}:=\mu_{2}+i \mu_{3}: M \rightarrow \mathfrak{k}^{*} \otimes_{\mathbb{R}} \mathbb{C}=\mathfrak{g}^{*},
$$

which turns out to be the moment map for the Hamiltonian $G$-action on $M$. Now assume that this $G$-action is free. The inclusion $\mu_{\mathrm{HK}}^{-1}(0) \subseteq \mu_{\mathbb{C}}^{-1}(0)$ then induces a map

$$
\varphi: M / / K \rightarrow M / / G
$$

where we recall that $M / / G$ is defined via (2). This map defines a diffeomorphism from $M / / / K$ to its image, the open subset $\left(G \cdot \mu_{\mathrm{HK}}^{-1}(0)\right) / G$ of $\mu_{\mathbb{C}}^{-1}(0) / G=M / / G$. Furthermore, $\varphi$ is an embedding of 
holomorphic symplectic manifolds with respect to the underlying holomorphic symplectic structure on $M / / K$.

\section{The HYPERKÄHLER GeOMETRY OF $T^{*}(G / H)$}

It will be convenient to standardize some of the Lie-theoretic notation used in this paper. Let $K$ be a compact connected semisimple Lie group, and fix a closed subgroup $L \subseteq K$. We will also let $G:=K_{\mathbb{C}}$ and $H:=L_{\mathbb{C}}$ denote the complexifications of $K$ and $L$, respectively, noting that $H$ is a closed reductive subgroup of $G$. Let $\mathfrak{k}, \mathfrak{l}, \mathfrak{g}$, and $\mathfrak{h}$ be the Lie algebras of $K, L$, $G$, and $H$, respectively, so that $\mathfrak{g}=\mathfrak{k} \otimes_{\mathbb{R}} \mathbb{C}$ and $\mathfrak{h}=\mathfrak{l} \otimes_{\mathbb{R}} \mathbb{C}$. Each of these Lie algebras comes equipped with the adjoint representation of the corresponding group, e.g. Ad : $G \rightarrow \mathrm{GL}(\mathfrak{g}), g \mapsto \operatorname{Ad}_{g}$. The symbol "Ad" will be used for all of the aforementioned adjoint representations, as context will always clarify any ambiguities that this abuse of notation may cause.

Let $\langle\cdot, \cdot\rangle: \mathfrak{g} \otimes_{\mathbb{C}} \mathfrak{g} \rightarrow \mathbb{C}$ denote the Killing form on $\mathfrak{g}$, which is $G$-invariant and non-degenerate. It follows that

$$
\mathfrak{g} \rightarrow \mathfrak{g}^{*}, \quad x \mapsto x^{\vee}:=\langle x, \cdot\rangle, \quad x \in \mathfrak{g}
$$

defines an isomorphism between the adjoint and coadjoint representations of $G$. With this in mind, we will sometimes take the moment map for a Hamiltonian $G$-action to be $\mathfrak{g}$-valued.

3.1. The cotangent bundle of $G$. Note that left and right multiplication give the commuting actions

$$
\begin{aligned}
& g \cdot h:=g h, \quad g, h \in G \\
& g \cdot h:=h g^{-1}, \quad g, h \in G
\end{aligned}
$$

of $G$ on itself, and that these lift to commuting Hamiltonian actions of $G$ on $T^{*} G$. To be more explicit about this point, we shall use the left trivialization of $T^{*} G$ and the Killing form to identify $T^{*} G$ with $G \times \mathfrak{g}$. The lifts of (6a) and (6b) then become

$$
\begin{aligned}
& g \cdot(h, x)=(g h, x), \quad g \in G,(h, x) \in G \times \mathfrak{g}, \\
& g \cdot(h, x)=\left(h g^{-1}, \operatorname{Ad}_{g}(x)\right), \quad g \in G,(h, x) \in G \times \mathfrak{g},
\end{aligned}
$$

respectively, while the induced symplectic form on $G \times \mathfrak{g}$ is defined on each tangent space $T_{(g, x)}(G \times$ $\mathfrak{g})=T_{g} G \oplus \mathfrak{g}$ as follows (see [26, Section 5, Equation (14L)]):

$$
\left(\Omega_{L}\right)_{(g, x)}\left(\left(d_{e} L_{g}\left(y_{1}\right), z_{1}\right),\left(d_{e} L_{g}\left(y_{2}\right), z_{2}\right)\right)=\left\langle y_{1}, z_{2}\right\rangle-\left\langle y_{2}, z_{1}\right\rangle+\left\langle x,\left[y_{1}, y_{2}\right]\right\rangle, \quad y_{1}, y_{2}, z_{1}, z_{2} \in \mathfrak{g}
$$

where $L_{g}: G \rightarrow G$ denotes left multiplication by $g$ and $d_{e} L_{g}: \mathfrak{g} \rightarrow T_{g} G$ is the differential of $L_{g}$ at the identity $e \in G$ (see. One can then verify that

$$
\begin{aligned}
\phi_{L}: G \times \mathfrak{g} \rightarrow \mathfrak{g}, & (g, x) \mapsto \operatorname{Ad}_{g}(x), \quad(g, x) \in G \times \mathfrak{g}, \\
\phi_{R}: G \times \mathfrak{g} \rightarrow \mathfrak{g}, & (g, x) \mapsto-x, \quad(g, x) \in G \times \mathfrak{g}
\end{aligned}
$$

are moment maps for (7a) and (7b) , respectively.

3.2. Kronheimer's hyperkähler structure on $T^{*} G$. Let $\mathbb{H}$ denote the quaternions, to be identified as a vector space with $\mathbb{R}^{4}$ via the usual basis $\{1, i, j, k\}$. Now consider the real vector space $C^{\infty}([0,1], \mathfrak{k})$ of all smooth maps $[0,1] \rightarrow \mathfrak{k}$. A choice of $K$-invariant inner product $\langle\cdot, \cdot\rangle_{\mathfrak{k}}$ on $\mathfrak{k}$ makes $\mathcal{M}:=C^{\infty}([0,1], \mathfrak{k}) \otimes_{\mathbb{R}} \mathbb{H}=C^{\infty}([0,1], \mathfrak{k})^{\oplus 4}$ into a Banach space with an infinite-dimensional hyperkähler manifold structure. This space carries the following hyperkähler structure-preserving action of $\mathcal{G}:=C^{\infty}([0,1], K)$, the gauge group of smooth maps $[0,1] \rightarrow K$ with pointwise multiplication 
as the group operation:

(10)

$$
\gamma \cdot\left(T_{0}, T_{1}, T_{2}, T_{3}\right):=\left(\operatorname{Ad}_{\gamma}\left(T_{0}\right)-\theta_{R}(\dot{\gamma}), \operatorname{Ad}_{\gamma}\left(T_{1}\right), \operatorname{Ad}_{\gamma}\left(T_{2}\right), \operatorname{Ad}_{\gamma}\left(T_{3}\right)\right), \quad \gamma \in \mathcal{G},\left(T_{0}, T_{1}, T_{2}, T_{3}\right) \in \mathcal{M},
$$

where $\theta_{R} \in \Omega^{1}(K ; \mathfrak{k})$ is the right-invariant Mauer-Cartan form on $K$. The subgroup

$$
\mathcal{G}_{0}:=\{\gamma \in \mathcal{G}: \gamma(0)=e=\gamma(1)\} \subseteq \mathcal{G}
$$

then acts freely on $\mathcal{M}$ with a hyperkähler moment map that can be written in the form $\Phi: \mathcal{M} \rightarrow$ $C^{\infty}([0,1], \mathfrak{k})^{\oplus 3}$. It turns out that $\Phi^{-1}(0)$ consists of the solutions to $N a ̈ h m$ 's equations (as defined in [10, Proposition 1], for example), and that Kronheimer constructed an explicit diffeomorphism

$$
G \times \mathfrak{g} \cong \mathcal{M} / / / \mathcal{G}_{0}=\Phi^{-1}(0) / \mathcal{G}_{0}
$$

(cf. [22, Proposition 1]). The smooth manifold $G \times \mathfrak{g}$ thereby inherits a hyperkähler structure $\left(I_{\ell}, \omega_{\ell}, b\right)$, $\ell=1,2,3$. We note that $\omega_{2}+i \omega_{3}$ equals the form $\Omega_{L}$ from (8), while $I_{1}$ is the usual complex structure on $G \times \mathfrak{g}$ (see [22, Section 2]).

Kronheimer's diffeomorphism 11 has some important equivariance properties that we now discuss. Note that $\mathcal{G}_{0}$ is the kernel of

$$
\mathcal{G} \rightarrow K \times K, \quad \gamma \mapsto(\gamma(0), \gamma(1)), \quad \gamma \in \mathcal{G},
$$

so that we may identify $\mathcal{G} / \mathcal{G}_{0}$ and $K \times K$ as Lie groups. The $\mathcal{G}$-action on $\mathcal{M}$ induces a residual action of $\mathcal{G} / \mathcal{G}_{0}=K \times K$ on $\mathcal{M} / / / \mathcal{G}_{0}$, and this residual action is known to be tri-Hamiltonian (see [10, Lemma 2]). Under (11), the action of $K=\{e\} \times K \subseteq K \times K$ on $\mathcal{M} / / / \mathcal{G}_{0}$ corresponds to the $K$-action (7a) on $G \times \mathfrak{g}$. The diffeomorphism also intertwines the action of $K=K \times\{e\} \subseteq K \times K$ on $M / / / \mathcal{G}_{0}$ with the $K$-action $7 \mathrm{~b}$ on $G \times \mathfrak{g}$.

The group $\mathrm{SO}_{3}(\mathbb{R})$ also has a natural manifestation in our setup. Given a point $\left(T_{0}, T_{1}, T_{2}, T_{3}\right) \in$ $\mathcal{M}=C^{\infty}([0,1], \mathfrak{k})^{\oplus 4}$ and a matrix $A=\left(a_{p q}\right) \in \mathrm{SO}_{3}(\mathbb{R})$, let us set

$$
T_{p}^{\prime}:=\sum_{q=1}^{3} a_{p q} T_{q}, \quad p=1,2,3 \quad \text { and } \quad A \cdot\left(T_{0}, T_{1}, T_{2}, T_{3}\right):=\left(T_{0}, T_{1}^{\prime}, T_{2}^{\prime}, T_{3}^{\prime}\right) .
$$

This action of $\mathrm{SO}_{3}(\mathbb{R})$ on $\mathcal{M}$ descends to an isometric action on the hyperkähler quotient $\mathcal{M} / / / \mathcal{G}_{0}$. One can use (11) to interpret this as an isometric action of $\mathrm{SO}_{3}(\mathbb{R})$ on the hyperkähler manifold $G \times \mathfrak{g}$, and it is not difficult to check that this action commutes with the $K$-actions (7a) and (7b). It is important to note that $\mathrm{SO}_{3}(\mathbb{R})$ does not preserve all of the hyperkähler structure on $G \times \mathfrak{g}$, in contrast to the $K$-actions. However, one can find a circle subgroup of $\mathrm{SO}_{3}(\mathbb{R})$ that preserves the Kähler triple $\left(I_{3}, \omega_{3}, b\right)$ on $G \times \mathfrak{g}$. A more explicit statement is that one can find an element $\theta \in \mathfrak{s o}_{3}(\mathbb{R})$ whose fundamental vector field $\tilde{\theta}$ on $G \times \mathfrak{g}$ satisfies the following properties: $\mathcal{L}_{\tilde{\theta}} \omega_{1}=\omega_{2}, \mathcal{L}_{\tilde{\theta}} \omega_{2}=-\omega_{1}$, and $\tilde{\theta}$ generates a circle action on $G \times \mathfrak{g}$ that preserves $\left(I_{3}, \omega_{3}, b\right)$. This circle subgroup acts by rotations on $\operatorname{span}_{\mathbb{R}}\left\{\omega_{1}, \omega_{2}\right\}$, and the following is (the $\theta$-component of) a moment map for its Hamiltonian action on $\left(G \times \mathfrak{g}, \omega_{3}\right)$ :

$$
\rho: G \times \mathfrak{g} \rightarrow \mathbb{R}, \quad\left[\left(T_{0}, T_{1}, T_{2}, T_{3}\right)\right] \mapsto \frac{1}{2} \int_{0}^{1}\left(\left\langle T_{1}, T_{1}\right\rangle_{\mathfrak{k}}+\left\langle T_{2}, T_{2}\right\rangle_{\mathfrak{k}}\right) d t
$$

where $\left[\left(T_{0}, T_{1}, T_{2}, T_{3}\right)\right]$ denotes the point in the $\Phi^{-1}(0) / \mathcal{G}_{0} \cong G \times \mathfrak{g}$ represented by $\left(T_{0}, T_{1}, T_{2}, T_{3}\right) \in$ $\Phi^{-1}(0)$ (see [10, Section 4]). This leads to the following lemma.

Lemma 2. The function $\rho$ is invariant under each of the $K$-actions (7a) and (7b) on $G \times \mathfrak{g}$.

Proof. Since $\langle\cdot, \cdot\rangle_{\mathfrak{k}}$ is a $K$-invariant inner product, the function

$$
\mathcal{M} \rightarrow \mathbb{R}, \quad\left(T_{0}, T_{1}, T_{2}, T_{3}\right) \mapsto \frac{1}{2} \int_{0}^{1}\left(\left\langle T_{1}, T_{1}\right\rangle_{\mathfrak{k}}+\left\langle T_{2}, T_{2}\right\rangle_{\mathfrak{k}}\right) d t
$$


is invariant under the action (10) of $\mathcal{G}$. This function therefore descends to a $\mathcal{G} / \mathcal{G}_{0}$-invariant function on the hyperkähler quotient $\mathcal{M} / / / \mathcal{G}_{0}$. The descended function is exactly $\rho$ once we identify $\mathcal{M} / / / \mathcal{G}_{0}$ with $G \times \mathfrak{g}$ via 11. Now recall that the $\mathcal{G} / \mathcal{G}_{0}$-action on $\mathcal{M} / / / \mathcal{G}_{0}$ corresponds to a $(K \times K)$-action on $G \times \mathfrak{g}$, meaning that $\rho$ is a $(K \times K)$-invariant function on $G \times \mathfrak{g}$. It just remains to recall that the $K$-action (7a) (resp. (7b) ) is the action of $K=\{e\} \times K \subseteq K \times K$ (resp. $K=K \times\{e\} \subseteq K \times K$ ).

3.3. The hyperkähler structure on $T^{*}(G / H)$. Let $G$ act on $G / H$ via left multiplication, and consider the canonical lift to a Hamiltonian action of $G$ on $T^{*}(G / H)$. Note also that $(\mathfrak{g} / \mathfrak{h})^{*}$ is a representation of $H$, and let $G \times{ }_{H}(\mathfrak{g} / \mathfrak{h})^{*}$ denote the quotient of $G \times(\mathfrak{g} / \mathfrak{h})^{*}$ by the following action of $H$ :

$$
h \cdot(g, \phi):=\left(g h^{-1}, h \cdot \phi\right) \quad h \in G,(g, \phi) \in G \times(\mathfrak{g} / \mathfrak{h})^{*} .
$$

We then have a canonical $G$-equivariant isomorphism $T^{*}(G / H) \cong G \times_{H}(\mathfrak{g} / \mathfrak{h})^{*}$, where $G$ acts on the latter variety via left multiplication on the first factor. At the same time, the $H$-representation $(\mathfrak{g} / \mathfrak{h})^{*}$ is canonically isomorphic to the annihilator $\mathfrak{h}^{\perp} \subseteq \mathfrak{g}$ of $\mathfrak{h}$ under the Killing form. We thus have a $G$-equivariant isomorphism

$$
T^{*}(G / H) \cong G \times_{H} \mathfrak{h}^{\perp},
$$

with $G \times{ }_{H} \mathfrak{h}^{\perp}$ defined analogously to $G \times{ }_{H}(\mathfrak{g} / \mathfrak{h})^{*}$.

Now consider the restriction of (7b) to an action of $H \subseteq G$ on $G \times \mathfrak{g}$, noting that this restricted action is Hamiltonian with respect to $\Omega_{L}$. The moment map for this $H$-action is obtained by composing the $\mathfrak{g}^{*}$-valued version of $\phi_{R}: G \times \mathfrak{g} \rightarrow \mathfrak{g}$ with the projection $\mathfrak{g}^{*} \rightarrow \mathfrak{h}^{*}$. It follows that the preimage of 0 under the new moment map is $G \times \mathfrak{h}^{\perp} \subseteq G \times \mathfrak{g}$. The symplectic quotient of $G \times \mathfrak{g}$ by $H$ is therefore given by

$$
(G \times \mathfrak{g}) / / H=G \times{ }_{H} \mathfrak{h}^{\perp} .
$$

It is straightforward to verify that the induced symplectic structure on $G \times{ }_{H} \mathfrak{h}^{\perp}$ renders (13) a $G$-equivariant isomorphism of symplectic varieites. It is also straightforward to check that

$$
\nu_{H}: G \times_{H} \mathfrak{h}^{\perp} \rightarrow \mathfrak{g}, \quad[(g, x)] \mapsto \operatorname{Ad}_{g}(x), \quad(g, x) \in G \times \mathfrak{h}^{\perp}
$$

is a moment map for the Hamiltonian action of $G$ on $G \times_{H} \mathfrak{h}^{\perp}$.

The above-defined holomorphic symplectic structure and Hamiltonian $G$-action on $G \times_{H} \mathfrak{h}^{\perp}$ turn out to come from a $(G, K)$-hyperkähler variety structure (see Definition 1), which we now discuss. Accordingly, recall that (7a) and (7b) define commuting, tri-Hamiltonian actions of $K$ on $G \times \mathfrak{g}$. Let us restrict the latter action to the subgroup $L \subseteq K$ fixed in the introduction to Section 3 , and then consider the associated hyperkähler quotient $(G \times \mathfrak{g}) / / / L$. Note that (7a) then descends to a tri-Hamiltonian action of $K$ on $(G \times \mathfrak{g}) / / / L$. At the same time, (44) takes the form of a $K$-equivariant map

$$
(G \times \mathfrak{g}) / / L \rightarrow(G \times \mathfrak{g}) / / H=G \times{ }_{H} \mathfrak{h}^{\perp} .
$$

One can then invoke [10, Section 2] and/or [27, Theorem 3.1] to deduce the following fact.

Theorem 3. The map (15) is a K-equivariant isomorphism of holomorphic symplectic manifolds.

Let $\left(I_{\ell}^{H}, \omega_{\ell}^{H}, b^{H}\right), \ell=1,2,3$, denote the hyperkähler manifold structure on $G \times{ }_{H} \mathfrak{h}^{\perp}$ for which (15) is an isomorphism of hyperkähler manifolds, which by the preceding discussion makes $G \times{ }_{H} \mathfrak{h}^{\perp}$ into a $(G, K)$-hyperkähler variety. To help investigate this $(G, K)$-hyperkähler structure, we use Lemma 2 to see that $\rho$ descends to a $K$-invariant function $\rho^{H}:(G \times \mathfrak{g}) / / L \rightarrow \mathbb{R}$. Note that since (15) is $K$-equivariant, we may regard $\rho^{H}$ as a $K$-invariant function on $G \times{ }_{H} \mathfrak{h}^{\perp}$.

Proposition 4. The function $\rho^{H}: G \times_{H} \mathfrak{h}^{\perp} \rightarrow \mathbb{R}$ is a K-invariant potential for the Kähler manifold $\left(G \times{ }_{H} \mathfrak{h}^{\perp}, I_{1}^{H}, \omega_{1}^{H}, b^{H}\right)$, i.e. $\omega_{1}^{H}=2 i \partial \bar{\partial} \rho^{H}$ for the Dolbeault operators $\partial$ and $\bar{\partial}$ associated with $I_{1}^{H}$. 
Proof. Let $\left(\mu_{1}, \mu_{2}, \mu_{3}\right): G \times \mathfrak{g} \rightarrow\left(\mathfrak{k}^{*}\right)^{\oplus 3}$ denote the hyperkähler moment map for the tri-Hamiltonian $K$-action (7b), and let $\left(\mu_{1}^{H}, \mu_{2}^{H}, \mu_{3}^{H}\right): G \times \mathfrak{g} \rightarrow\left(\mathfrak{l}^{*}\right)^{\oplus 3}$ be the induced hyperkähler moment map for the action of $L \subseteq K$. Consider the action of $\mathrm{SO}_{3}(\mathbb{R})$ on $G \times \mathfrak{g}$, recalling our description of a specific subgroup $S^{1} \subseteq \mathrm{SO}_{3}(\mathbb{R})$ and its action on $G \times \mathfrak{g}$ (see 3.2). This description implies that $S^{1}$ preserves $\mu_{3}$ and acts by rotations on $\operatorname{span}_{\mathbb{R}}\left\{\mu_{1}, \mu_{2}\right\}$. We conclude that $S^{1}$ preserves $\mu_{3}^{H}$ and acts by rotations on $\operatorname{span}_{\mathbb{R}}\left\{\mu_{1}^{H}, \mu_{2}^{H}\right\}$, so that the submanifold $\left(\mu_{1}^{H}\right)^{-1}(0) \cap\left(\mu_{2}^{H}\right)^{-1}(0) \cap\left(\mu_{3}^{H}\right)^{-1}(0) \subseteq G \times \mathfrak{g}$ is necessarily $S^{1}$-invariant. Observe that the actions of $S^{1}$ and $L$ on this submanifold commute, owing to the fact that the action of $\mathrm{SO}_{3}(\mathbb{R})$ on $G \times \mathfrak{g}$ commutes with the $K$-action (7b). The quotient

$$
\left(\left(\mu_{1}^{H}\right)^{-1}(0) \cap\left(\mu_{2}^{H}\right)^{-1}(0) \cap\left(\mu_{3}^{H}\right)^{-1}(0)\right) / L=(G \times \mathfrak{g}) / / L
$$

therefore carries a residual $S^{1}$-action, so that we may use the hyperkähler isomorphism (15) to equip $G \times{ }_{H} \mathfrak{h}^{\perp}$ with a corresponding $S^{1}$-action. The relations (3) then imply that $S^{1}$ preserves $\omega_{3}^{H}$. Now consider the element $\theta \in \mathfrak{s o}_{3}(\mathbb{R})$ discussed in 3.2 , recalling that $\rho$ is the $\theta$-component of a moment map for the $S^{1}$-action on $G \times \mathfrak{g}$. It is then straightforward to check that $\rho^{H}$ is the $\theta$-component of a moment map for the $S^{1}$-action that preserves $\omega_{3}^{H}$. Note also that the identities $\mathcal{L}_{\tilde{\theta}} \omega_{1}=\omega_{2}$ and $\mathcal{L}_{\tilde{\theta}} \omega_{2}=-\omega_{1}$ give

$$
\mathcal{L} \tilde{\tilde{\theta}} \omega_{1}^{H}=\omega_{2}^{H} \quad \text { and } \quad \mathcal{L}_{\tilde{\tilde{\theta}}} \omega_{2}^{H}=-\omega_{1}^{H},
$$

where $\tilde{\tilde{\theta}}$ is the fundamental vector field on $G \times_{H} \mathfrak{h}^{\perp}$ associated to $\theta$. These last two sentences give exactly the ingredients needed to reproduce a calculation from [15, Section 3(E)], to the effect that $\rho^{H}$ is a Kähler potential for $\left(I_{1}^{H}, \omega_{1}^{H}, b^{H}\right)$.

\section{THE HYPERKÄHLER SLICE CONSTRUCTION}

4.1. The slice as a symplectic variety. Recall the notation established in the introduction to Section 3 , and let

$$
\operatorname{ad}: \mathfrak{g} \rightarrow \mathfrak{g l}(\mathfrak{g}), \quad x \mapsto \operatorname{ad}_{x}, \quad x \in \mathfrak{g}
$$

denote the adjoint representation of $\mathfrak{g}$. One calls $\tau=(\xi, h, \eta) \in \mathfrak{g}^{\oplus 3}$ an $\mathfrak{s l}_{2}$-triple if $[\xi, \eta]=h$, $[h, \xi]=2 \xi$, and $[h, \eta]=-2 \eta$, in which case there is an associated Slodowy slice

$$
S_{\tau}:=\xi+\operatorname{ker}\left(\operatorname{ad}_{\eta}\right) \subseteq \mathfrak{g} .
$$

We will make extensive use of the affine variety $G \times S_{\tau}$, some geometric features of which we now develop.

Consider the isomorphisms $T^{*} G \cong G \times \mathfrak{g}^{*} \cong G \times \mathfrak{g}$ induced by the right trivialization of $T^{*} G$ and the Killing form. The symplectic form on $T^{*} G$ thereby corresponds to such a form $\Omega_{R}$ on $G \times \mathfrak{g}$, described as follows on the tangent space $T_{(g, x)}(G \times \mathfrak{g})=T_{g} G \oplus \mathfrak{g}$ (see [26, Section 5, Equation (14R)]:

$$
\left(\Omega_{R}\right)_{(g, x)}\left(\left(d_{e} R_{g}\left(y_{1}\right), z_{1}\right),\left(d_{e} R_{g}\left(y_{2}\right), z_{2}\right)\right)=\left\langle y_{1}, z_{2}\right\rangle-\left\langle y_{2}, z_{1}\right\rangle-\left\langle x,\left[y_{1}, y_{2}\right]\right\rangle
$$

for all $y_{1}, y_{2}, z_{1}, z_{2} \in \mathfrak{g}$, where $R_{g}: G \rightarrow G$ is right multiplication by $g, d_{e} R_{g}$ is its differential at $e$. It turns out that $G \times S_{\tau}$ is a symplectic subvariety of $\left(G \times \mathfrak{g}, \Omega_{R}\right)$. The $G$-action

$$
g \cdot(h, x)=\left(h g^{-1}, x\right), \quad g \in G,(h, x) \in G \times S_{\tau}
$$

is then Hamiltonian and

$$
\mu_{\tau}: G \times S_{\tau} \rightarrow \mathfrak{g}, \quad(g, x) \mapsto-\operatorname{Ad}_{g^{-1}}(x), \quad(g, x) \in G \times S_{\tau}
$$

is a moment map. 
Remark 5. Bielawski's paper [4] uses $\Omega_{R}$ to realize $G \times S_{\tau}$ as a symplectic subvariety $G \times \mathfrak{g}$, as opposed to using the other symplectic form $\Omega_{L}$ (see (8)). It is for the sake of consistency with Bielawski's work that we are using the same convention. However, this is the only case in which we use $\Omega_{R}$ preferentially to $\Omega_{L}$.

Now let $X$ be a symplectic variety endowed with a Hamiltonian $G$-action and moment map $\mu$ : $X \rightarrow \mathfrak{g}$. The diagonal action of $G$ on $X \times\left(G \times S_{\tau}\right)$ is then Hamiltonian and admits a moment map of

$$
\tilde{\mu}: X \times\left(G \times S_{\tau}\right) \rightarrow \mathfrak{g}, \quad(x,(g, y)) \mapsto \mu(x)+\mu_{\tau}(g, y), \quad x \in X,(g, y) \in G \times S_{\tau} .
$$

Noting that this diagonal action is free, one has the symplectic quotient

$$
\left(X \times\left(G \times S_{\tau}\right)\right) / / G=\tilde{\mu}^{-1}(0) / G .
$$

Proposition 6. Let $(X, \omega)$ be a symplectic variety on which $G$ acts in a Hamiltonian fashion with moment map $\mu: X \rightarrow \mathfrak{g}$, and let $\tau$ be an $\mathfrak{s l}_{2}$-triple. The following statements then hold.

(i) There is a canonical isomorphism of affine varieties $\mu^{-1}\left(S_{\tau}\right) \cong\left(X \times\left(G \times S_{\tau}\right)\right) / / G$.

(ii) Under the isomorphism from (i), the symplectic form on $\left(X \times\left(G \times S_{\tau}\right)\right) / / G$ corresponds to the restriction of $\omega$ to $\mu^{-1}\left(S_{\tau}\right)$.

(iii) $\mu^{-1}\left(S_{\tau}\right)$ is a symplectic subvariety of $X$.

Proof. To prove (i), note that $(x,(e, \mu(x))) \in \tilde{\mu}^{-1}(0)$ for all $x \in \mu^{-1}\left(S_{\tau}\right)$. We may therefore consider the morphism

$$
\varphi: \mu^{-1}\left(S_{\tau}\right) \rightarrow \tilde{\mu}^{-1}(0), \quad x \mapsto(x,(e, \mu(x))), \quad x \in \mu^{-1}\left(S_{\tau}\right),
$$

and its composition with the quotient map $\pi: \tilde{\mu}^{-1}(0) \rightarrow \tilde{\mu}^{-1}(0) / G=\left(X \times\left(G \times S_{\tau}\right)\right) / / G$, i.e.

$$
\bar{\varphi}: \mu^{-1}\left(S_{\tau}\right) \rightarrow\left(X \times\left(G \times S_{\tau}\right)\right) / / G, \quad x \mapsto[(x,(e, \mu(x)))], \quad x \in \mu^{-1}\left(S_{\tau}\right) .
$$

At the same time, it is straightforward to check that $g \cdot x \in \mu^{-1}\left(S_{\tau}\right)$ for all $(x,(g, y)) \in \tilde{\mu}^{-1}(0)$. We thus have the morphism

$$
\psi: \tilde{\mu}^{-1}(0) \rightarrow \mu^{-1}\left(S_{\tau}\right), \quad(x,(g, y)) \mapsto g \cdot x, \quad(x,(g, y)) \in \tilde{\mu}^{-1}(0),
$$

which is easily seen to be $G$-invariant. It follows that $\psi$ descends to the quotient $\tilde{\mu}^{-1}(0) / G=$ $\left(X \times\left(G \times S_{\tau}\right)\right) / / G$, thereby giving a morphism $\bar{\psi}:\left(X \times\left(G \times S_{\tau}\right)\right) / / G \rightarrow \mu^{-1}\left(S_{\tau}\right)$. Furthermore, it is a straightforward calculation that $\bar{\varphi}$ and $\bar{\psi}$ are inverses. This proves (i).

In preparation for (ii), let $\bar{\omega}$ denote the symplectic form on $\left(X \times\left(G \times S_{\tau}\right)\right) / / G$ and consider the inclusions $j: G \times S_{\tau} \rightarrow G \times \mathfrak{g}$ and $k: \tilde{\mu}^{-1}(0) \rightarrow X \times\left(G \times S_{\tau}\right)$. Note that $\pi^{*}(\bar{\omega})$ is the restriction to $\tilde{\mu}^{-1}(0)$ of the symplectic form on $X \times\left(G \times S_{\tau}\right)$. This last symplectic form is $\omega \oplus j^{*}\left(\Omega_{R}\right)$, so that we have

$$
\pi^{*}(\bar{\omega})=k^{*}\left(\omega \oplus j^{*}\left(\Omega_{R}\right)\right)
$$

Our objective is to prove that $\bar{\varphi}^{*}(\bar{\omega})=\ell^{*}(\omega)$, where $\ell: \mu^{-1}\left(S_{\tau}\right) \rightarrow X$ is the inclusion. Accordingly, note that

$$
\begin{array}{rlrl}
\bar{\varphi}^{*}(\bar{\omega}) & =\varphi^{*}\left(\pi^{*}(\bar{\varphi})\right) & & {[\text { since } \bar{\varphi}=\pi \circ \varphi]} \\
& =(k \circ \varphi)^{*}\left(\omega \oplus j^{*}\left(\Omega_{R}\right)\right) & {[\text { by (18) }] .}
\end{array}
$$

It follows that

$$
\left(\bar{\varphi}^{*}(\bar{\omega})\right)_{x}\left(v_{1}, v_{2}\right)=\left(\omega_{x} \oplus\left(\Omega_{R}\right)_{(e, \mu(x))}\right)\left(d_{x} \varphi\left(v_{1}\right), d_{x} \varphi\left(v_{2}\right)\right)
$$

for all $x \in \mu^{-1}\left(S_{\tau}\right)$ and $v_{1}, v_{2} \in T_{x}\left(\mu^{-1}\left(S_{\tau}\right)\right)$. At the same time, (17) implies the identity

$$
d_{x} \varphi\left(v_{i}\right)=\left(v_{i},\left(0, d_{x} \mu\left(v_{i}\right)\right)\right)
$$


for $i=1,2$ in the tangent space

$$
T_{(x,(e, \mu(x)))}\left(\tilde{\mu}^{-1}(0)\right) \subseteq T_{(x,(e, \mu(x)))}\left(X \times\left(G \times S_{\tau}\right)\right)=T_{x} X \oplus\left(\mathfrak{g} \oplus T_{\mu(x)} S_{\tau}\right) .
$$

By incorporating this into (19), we obtain

$$
\begin{aligned}
\left(\bar{\varphi}^{*}(\bar{\omega})\right)_{x}\left(v_{1}, v_{2}\right) & =\omega_{x}\left(v_{1}, v_{2}\right)+\left(\Omega_{R}\right)_{(e, \mu(x))}\left(\left(0, d_{x} \mu\left(v_{1}\right)\right),\left(0, d_{x} \mu\left(v_{2}\right)\right)\right. \\
& =\omega_{x}\left(v_{1}, v_{2}\right)
\end{aligned}
$$

We conclude that $\bar{\varphi}^{*}(\bar{\omega})=\ell^{*}(\omega)$, proving (ii).

It remains only to prove (iii), i.e. the claim that $\ell^{*}(\omega)$ is non-degenerate. However, this follows immediately from (i), (ii), and the fact that $\bar{\omega}$ is non-degenerate.

4.2. Bielawski's construction. We now review the pertinent hyperkähler-geometric features of $\mu^{-1}\left(S_{\tau}\right)$, which are largely due to Bielawski's work [4]. The following $(G, K)$-hyperkähler variety will play an essential role.

Theorem 7. If $\tau$ is an $\mathfrak{s l}_{2}$-triple, then $G \times S_{\tau}$ is canonically a $(G, K)$-hyperkähler variety. The Hamiltonian $G$-action and underlying holomorphic symplectic structure on $G \times S_{\tau}$ associated with this $(G, K)$-hyperkähler structure are precisely those described in 4.1.

Now let $X$ be any $(G, K)$-hyperkähler variety. Given an $\mathfrak{s l}_{2}$-triple $\tau$, note that product manifold $X \times\left(G \times S_{\tau}\right)$ is naturally hyperkähler and carries a free, diagonal $G$-action. It is then not difficult to check that $X \times\left(G \times S_{\tau}\right)$ is a $(G, K)$-hyperkähler variety, with underlying holomorphic symplectic structure equal to the natural product holomorphic symplectic structure on $X \times\left(G \times S_{\tau}\right)$. With this in mind, we can define hyperkähler slices as follows.

Definition 8. Given a $(G, K)$-hyperkähler variety $X$ and an $\mathfrak{s l}_{2}$-triple $\tau$, we refer to $\left(X \times\left(G \times S_{\tau}\right)\right) / / / K$ as the hyperkähler slice for $X$ and $\tau$.

This construction can be used to produce a number of well-studied hyperkähler manifolds, some of which are mentioned in the introduction of [5]. For several of these examples, there is a particularly concrete description of the underlying holomorphic symplectic manifold. Indeed, let $X$ and $\tau$ be as described in the definition above. Note that (4) manifests as a map

$$
\left(X \times\left(G \times S_{\tau}\right)\right) / / K \rightarrow\left(X \times\left(G \times S_{\tau}\right)\right) / / G,
$$

which features in the following rephrased version of [4, Theorem 1].

Theorem 9 (Bielawski). Let $\tau$ be an $\mathfrak{s l}_{2}$-triple, and let $\left(X,\left(I_{\ell}, \omega_{\ell}, b\right)_{\ell=1}^{3}\right)$ be a $(G, K)$-hyperkähler variety with complex moment map $\mu: X \rightarrow \mathfrak{g}$. Consider the map

$$
\left(X \times\left(G \times S_{\tau}\right)\right) / / K \rightarrow \mu^{-1}\left(S_{\tau}\right)
$$

obtained by composing (20) with the isomorphism $\left(X \times\left(G \times S_{\tau}\right)\right) / / G \stackrel{\cong}{\rightarrow} \mu^{-1}\left(S_{\tau}\right)$ from Proposition [6(i). If the Kähler manifold $\left(X, I_{1}, \omega_{1}, b\right)$ has a $K$-invariant potential that is bounded from below on each $G$-orbit, then (21) is an isomorphism of holomorphic symplectic manifolds.

Remark 10. Bielawski speaks of hyperkähler slices only when the hypotheses of Theorem 9 are satisfied (see [5, Section 1]). He then defines a hyperkähler slice to be a hyperkähler manifold of the form $\mu^{-1}\left(S_{\tau}\right)$, where $\mu^{-1}\left(S_{\tau}\right)$ is equipped with the hyperkähler structure induced through the isomorphism (21). In particular, Definition 8 mildly generalizes Bielawski's original notion.

Let us briefly consider the hyperkähler slice construction for $(G, K)$-hyperkähler varieties of the form $\left(G \times_{H} \mathfrak{h}^{\perp},\left(I_{\ell}^{H}, \omega_{\ell}^{H}, b^{H}\right)_{\ell=1}^{3}\right)$, as introduced in 3.3. Accordingly, recall the notation adopted in 3.3. The function $\rho^{H}$ is bounded from below on all of $G \times_{H} \mathfrak{h}^{\perp}$ (see (12)), while we recall that $\rho^{H}$ is 
a $K$-invariant potential for the Kähler manifold $\left(G \times_{H} \mathfrak{h}^{\perp},\left(I_{1}^{H}, \omega_{1}^{H}, b^{H}\right)\right)$ (see Proposition 44). It then follows from Theorem 9 that

$$
\left(\left(G \times{ }_{H} \mathfrak{h}^{\perp}\right) \times\left(G \times S_{\tau}\right)\right) / / K \cong \nu_{H}^{-1}\left(S_{\tau}\right)
$$

as holomorphic symplectic manifolds for all $\mathfrak{s l}_{2}$-triples $\tau$ in $\mathfrak{g}$. We exploit this fact in what follows.

4.3. The regular Slodowy slice. Recall that $\operatorname{dim}\left(\operatorname{ker}\left(\operatorname{ad}_{x}\right)\right) \geq r$ for all $x \in \mathfrak{g}$, and that $x$ is called regular if equality holds. Let $\mathfrak{g}_{\text {reg }} \subseteq \mathfrak{g}$ denote the set of all regular elements, which is known to be a $G$-invariant, open, dense subvariety of $\mathfrak{g}$. This leads to the notion of a regular $\mathfrak{s l}_{2}$-triple, i.e. an $\mathfrak{s l}_{2}$-triple $\tau=(\xi, h, \eta)$ in $\mathfrak{g}$ for which $\xi \in \mathfrak{g}_{\text {reg }}$. Fix one such triple $\tau$ for the duration of this paper, and let $S_{\text {reg }}:=S_{\tau}$ denote the associated Slodowy slice. The slice $S_{\text {reg }}$ is known to be contained in $\mathfrak{g}_{\text {reg }}$, and to be a fundamental domain for the action of $G$ on $\mathfrak{g}_{\text {reg }}$ (see [18, Theorem 8]). Note that this last sentence may be rephrased as follows: $x \in \mathfrak{g}$ belongs to $\mathfrak{g}_{\text {reg }}$ if and only if $x$ is $G$-conjugate to a point in $S_{\text {reg }}$, in which case $x$ is $G$-conjugate to a unique point in $S_{\text {reg }}$.

As discussed in the 1.2, we wish to study the emptiness problem for hyperkähler slices of the form $\left(\left(G \times{ }_{H} \mathfrak{h}^{\perp}\right) \times\left(G \times S_{\text {reg }}\right)\right) / / / K$. The following result is a crucial first step.

Proposition 11. The hyperkähler slice $\left(\left(G \times{ }_{H} \mathfrak{h}^{\perp}\right) \times\left(G \times S_{\text {reg }}\right)\right) / / / K$ is non-empty if and only if $\mathfrak{h}^{\perp} \cap \mathfrak{g}_{\mathrm{reg}} \neq \emptyset$.

Proof. Using (22), we conclude that $\left(\left(G \times_{H} \mathfrak{h}^{\perp}\right) \times\left(G \times S_{\text {reg }}\right)\right) / / K \neq \emptyset$ if and only if the image of

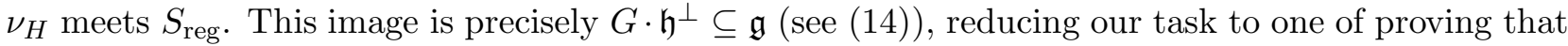
$G \cdot \mathfrak{h}^{\perp} \cap S_{\text {reg }} \neq \emptyset$ if and only if $\mathfrak{h}^{\perp} \cap \mathfrak{g}_{\text {reg }} \neq \emptyset$. To prove this, we simply appeal to the discussion of $S_{\text {reg }}$ above and note that $x \in \mathfrak{h}^{\perp}$ belongs to $\mathfrak{g}_{\text {reg }}$ if and only if $x$ is $G$-conjugate to a point in $S_{\text {reg. }}$.

\section{The SPherical GeOMEtry of $G / H$}

5.1. The image of the moment map. Let us continue with the notation set in the introduction of Section 3. Choose opposite Borel subgroups $B, B_{-} \subseteq G$, declaring the former to be the positive Borel and the latter to be the negative Borel. It follows that $T:=B \cap B_{-}$is a maximal torus of $G$, and we shall let $\mathfrak{b}, \mathfrak{b}_{-}$, and $\mathfrak{t}$ denote the Lie algebras of $B, B_{-}$, and $T$, respectively. We thus have a weight lattice $\Lambda \subseteq \mathfrak{t}^{*}$ and canonical group isomorphisms $\Lambda \cong \operatorname{Hom}\left(T, \mathbb{C}^{\times}\right) \cong \operatorname{Hom}\left(B, \mathbb{C}^{\times}\right)$, where Hom is taken in the category of algebraic groups. We also have sets of roots $\Delta \subseteq \Lambda$, positive roots $\Delta_{+} \subseteq \Delta$, negative roots $\Delta_{-} \subseteq \Delta$, and simple roots $\Pi \subseteq \Delta_{+}$. Note that by definition

$$
\mathfrak{b}=\mathfrak{t} \oplus \bigoplus_{\alpha \in \Delta_{+}} \mathfrak{g}_{\alpha} \quad \text { and } \quad \mathfrak{b}_{-}=\mathfrak{t} \oplus \bigoplus_{\alpha \in \Delta_{-}} \mathfrak{g}_{\alpha}
$$

where $\mathfrak{g}_{\alpha}$ is the root space associated to $\alpha \in \Delta$.

We now establish two important conventions. To this end, recall the isomorphism (5) between the adjoint and coadjoint representations of $G$. Our first convention is to use $(\cdot)^{\vee}$ for both (5) and its inverse, so that the inverse will presented as

$$
\mathfrak{g}^{*} \cong \mathfrak{g}, \quad \phi \mapsto \phi^{\vee}, \quad \phi \in \mathfrak{g}^{*} .
$$

As for our second convention, note that the map $\mathfrak{g}^{*} \rightarrow \mathfrak{t}^{*}$ restricts to an isomorphism from the image of $\mathfrak{t}$ under (5) to $\mathfrak{t}^{*}$. We will use this isomorphism to regard $\mathfrak{t}^{*}$ as belonging to $\mathfrak{g}^{*}$.

Now let $Y$ be a smooth, irreducible $G$-variety having field of rational functions $\mathbb{C}(Y)$, noting that $\mathbb{C}(Y)$ is then a $G$-module. A non-zero $f \in \mathbb{C}(Y)$ is called a $B$-semi-invariant rational function of weight $\lambda \in \Lambda$ if $b \cdot f=\lambda(b) f$ for all $b \in B$. Those $\lambda$ admitting such an $f$ form the weight lattice of $Y$, i.e.

$$
\Lambda_{Y}:=\{\lambda \in \Lambda: \exists \text { a } B \text {-semi-invariant rational function on } Y \text { of weight } \lambda\} .
$$


The weight lattice of $Y$ can also be viewed as the character lattice of a quotient of $T$, once we appeal to Knop's local structure theorem [34, Theorem 4.7]. This theorem gives a parabolic subgroup $P \subseteq G$ that contains $B$, has a Levi decomposition $P=P_{u} L$ with $T \subseteq L$, and satisfies the following property: there exists a locally closed affine $P$-stable subvariety $Z \subseteq Y$ such that $P_{u} \times Z \rightarrow Y$ maps surjectively onto an open affine subset $Y_{0}$ of Y. One also has $[L, L] \subseteq L_{0} \subseteq L$, where $L_{0}$ is the kernel of the $L$-action on $Z$. The quotient $A_{Y}:=L / L_{0}$ is a torus that acts freely on $Z$, and there exists an affine variety $C$ with a trivial $L$-action such that $Z \cong A_{Y} \times C$ as $L$-varieties. It follows that $\Lambda_{Y}=\operatorname{Hom}\left(A_{Y}, \mathbb{C}^{\times}\right)$.

The subspace $\mathfrak{a}_{Y}^{*}:=\Lambda_{Y} \otimes_{\mathbb{Z}} \mathbb{C} \subseteq \mathfrak{t}^{*}$ is sometimes called the Cartan space of the $G$-variety $Y$. Let $\Lambda_{Y}^{\vee} \subseteq \mathfrak{t}$ and $\mathfrak{a}_{Y} \subseteq \mathfrak{t}$ denote the preimage and image of $\Lambda_{Y}$ and $\mathfrak{a}_{Y}^{*}$ under (5), respectively, noting that

$$
\widetilde{A}_{Y}:=\Lambda_{Y}^{\vee} \otimes_{\mathbb{Z}} \mathbb{C}^{\times}
$$

is a subtorus of $T$ with Lie algebra $\mathfrak{a}_{Y}$. We shall also refer to $\mathfrak{a}_{Y}$ as the Cartan space of $Y$.

Example 12. In what follows, we compute the Cartan space of $G / T$. Let $\Lambda_{+} \subseteq \mathfrak{t}^{*}$ denote the set of dominant weights of $G$, and let $V_{\lambda}$ be the irreducible $G$-module of highest weight $\lambda \in \Lambda_{+}$. Recall the following classical fact about $\mathbb{C}[G / T]$, the coordinate ring of $G / T$ :

$$
\mathbb{C}[G / T] \cong \bigoplus_{\Lambda \in \Lambda_{+}}\left(V_{\lambda}^{*}\right)^{\oplus d_{\lambda}}
$$

as $G$-modules, where $d_{\lambda}:=\operatorname{dim}\left(\left(V_{\lambda}\right)^{T}\right)$ and $\left(V_{\lambda}\right)^{T}$ is the subspace of $T$-fixed vectors in $V_{\lambda}$. Note that $d_{\lambda} \neq 0$ if and only if $\lambda$ lies in the root lattice $Q \subseteq \mathfrak{t}^{*}$. Note also that $\left(V_{\lambda}\right)^{*} \cong V_{-w_{0} \lambda}$ as $G$-modules, where $w_{0}$ is the longest element of the Weyl group $W:=N_{G}(T) / T$. It follows that for $\lambda \in \Lambda_{+}, V_{\lambda}$ is an irreducible summand of $\mathbb{C}[G / T]$ if and only if $\lambda \in-w_{0}\left(\Lambda_{+} \cap Q\right)=\Lambda_{+} \cap Q$. Since $\mathbb{C}[G / T]$ is a $G$-submodule of $\mathbb{C}(G / T)$, this implies that $\Lambda_{+} \cap Q$ is contained in $\Lambda_{G / T}$. Now observe that $\Lambda_{+} \cap Q$ generates $\mathfrak{t}^{*}$ over $\mathbb{C}$, yielding $\mathfrak{a}_{G / T}^{*}=\Lambda_{G / T} \otimes_{\mathbb{Z}} \mathbb{C}=\mathfrak{t}^{*}$. We also conclude that $\mathfrak{a}_{G / T}=\mathfrak{t}$.

We now recall a key geometric feature of the Cartan space construction. Let $Y$ be any smooth, irreducible $G$-variety and consider the canonical lift of the $G$-action on $Y$ to a $G$-action on $T^{*} Y$. The latter action is Hamiltonian with respect to the standard symplectic form on $T^{*} Y$, and there is a distinguished moment map $\mu_{Y}: T^{*} Y \rightarrow \mathfrak{g}$. Lemma 3.1 and Corollary 3.3 from [17] then combine to give the following equality of closures in $\mathfrak{g}$.

Theorem 13 (Knop). If $Y$ is a smooth, irreducible, quasi-affine G-variety, then $\overline{\mu_{Y}\left(T^{*} Y\right)}=\overline{G \cdot \mathfrak{a}_{Y}}$.

5.2. a-regularity. Recall the notation set in the introduction to Section 3, which we now use together with the notation of [5.1. It is then not difficult to prove that $\mathfrak{a}_{G / H}$ depends only on the pair $(\mathfrak{g}, \mathfrak{h})$. For this reason, we set $\mathfrak{a}(\mathfrak{g}, \mathfrak{h})^{*}:=\mathfrak{a}_{G / H}^{*}$ and $\mathfrak{a}(\mathfrak{g}, \mathfrak{h}):=\mathfrak{a}_{G / H}$. We will sometimes denote $\mathfrak{a}(\mathfrak{g}, \mathfrak{h})$ (resp. $\left.\mathfrak{a}(\mathfrak{g}, \mathfrak{h})^{*}\right)$ by $\mathfrak{a}\left(\right.$ resp. $\left.\mathfrak{a}^{*}\right)$ when the underlying pair $(\mathfrak{g}, \mathfrak{h})$ is clear from context.

Definition 14. We say that the pair $(G, H)$ or the corresponding pair $(\mathfrak{g}, \mathfrak{h})$ of Lie algebras is $\mathfrak{a}$-regular if $\mathfrak{a}(\mathfrak{g}, \mathfrak{h})$ contains a regular element of $\mathfrak{g}$.

We now give a few characterizations of a-regularity. In what follows, $\widetilde{A}_{G / H}$ is the subtorus of $T$ defined by setting $Y=G / H$ in (23) and $Z_{G}\left(\widetilde{A}_{G / H}\right)$ consists of all $g \in G$ that commute with every element of $\widetilde{A}_{G / H}$. We also let $Z_{G}(\mathfrak{a})$ be the subgroup of all $g \in G$ that fix a pointwise, and we let $\mathfrak{z}_{\mathfrak{g}}(\mathfrak{a})$ be the subspace of all $x \in \mathfrak{g}$ that commute with every element of $\mathfrak{a}$.

Proposition 15. With all notation as described above, the following conditions are equivalent.

(i) $(G, H)$ is a-regular;

(ii) $\mathfrak{h}^{\perp} \cap \mathfrak{g}_{\text {reg }} \neq \emptyset$;

(iii) $Z_{G}(\mathfrak{a})=T$. 
Proof. We begin by proving that $\mathfrak{h}^{\perp} \cap \mathfrak{g}_{\text {reg }} \neq \emptyset$ if and only if $(G, H)$ is $\mathfrak{a}$-regular. To show the forward implication, assume that $\mathfrak{h}^{\perp} \cap \mathfrak{g}_{\text {reg }} \neq \emptyset$. Identifying $T^{*}(G / H)$ with $G \times{ }_{H} \mathfrak{h}^{\perp}$ and recalling the moment map $\nu_{H}$ (see (14)), Theorem 13 implies that

$$
\overline{\nu_{H}\left(G \times_{H} \mathfrak{h}^{\perp}\right)}=\overline{G \cdot \mathfrak{a}} .
$$

This amounts to the statement that

$$
\overline{G \cdot \mathfrak{h}^{\perp}}=\overline{G \cdot \mathfrak{a}} .
$$

Since $\mathfrak{h}^{\perp} \cap \mathfrak{g}_{\text {reg }} \neq \emptyset$ by hypothesis, we must have $\overline{G \cdot \mathfrak{a}} \cap \mathfrak{g}_{\text {reg }} \neq \emptyset$. Note also that $G \cdot \mathfrak{a}$ is a constructible subset of $\mathfrak{g}$, so that $G \cdot \mathfrak{a}$ intersects every non-empty open subset of $\overline{G \cdot \mathfrak{a}}$. These last two sentences imply that $G \cdot \mathfrak{a} \cap \mathfrak{g}_{\text {reg }} \neq \emptyset$, which is equivalent to $\mathfrak{a} \cap \mathfrak{g}_{\text {reg }} \neq \emptyset$. We conclude that $(G, H)$ is $\mathfrak{a}$-regular. In an analogous way, one argues that $(G, H)$ being $\mathfrak{a}$-regular implies $\mathfrak{h}^{\perp} \cap \mathfrak{g}_{\text {reg }} \neq \emptyset$.

We are reduced to establishing that $(G, H)$ is $\mathfrak{a}$-regular if and only if $Z_{G}(\mathfrak{a})=T$. Accordingly, recall that an element of $\mathfrak{t}$ is regular if and only if it does not lie on any root hyperplane. It follows that $(G, H)$ is not $\mathfrak{a}$-regular if and only if $\mathfrak{a}$ belongs to the union of all root hyperplanes. Since $\mathfrak{a}$ is irreducible, this is equivalent to $\mathfrak{a}$ being contained in a particular root hyperplane, i.e. $\mathfrak{a} \subseteq \operatorname{ker}(\alpha)$ for some $\alpha \in \Delta$. This holds if and only if $\mathfrak{g}_{\alpha} \subseteq \mathfrak{z}_{\mathfrak{g}}(\mathfrak{a})$ for some $\alpha \in \Delta$. Now note that $\mathfrak{z}_{\mathfrak{g}}(\mathfrak{a})$ is a $T$-invariant subspace of $\mathfrak{g}$ containing $\mathfrak{t}$, meaning that

$$
\mathfrak{z}_{\mathfrak{g}}(\mathfrak{a})=\mathfrak{t} \oplus \bigoplus_{\alpha \in S} \mathfrak{g}_{\alpha}
$$

for some subset $S \subseteq \Delta$. It follows that $\mathfrak{g}_{\alpha} \subseteq \mathfrak{z}_{\mathfrak{g}}(\mathfrak{a})$ for some $\alpha \in \Delta$ if and only if $\mathfrak{z}_{\mathfrak{g}}(\mathfrak{a}) \neq \mathfrak{t}$. The second of these conditions is equivalent to having $Z_{G}(\mathfrak{a}) \neq T$, if one knows $Z_{G}(\mathfrak{a})$ to be connected and have a Lie algebra of $\mathfrak{z} \mathfrak{g}(\mathfrak{a})$. Connectedness follows from the observation that $Z_{G}(\mathfrak{a})=Z_{G}\left(\widetilde{A}_{G / H}\right)$ (see [33, Theorem 24.4.8]), together with the fact that centralizers of tori are connected (see [33, Proposition 28.3.1]). At the same time, it is clear that $\mathfrak{z}_{\mathfrak{g}}(\mathfrak{a})$ is the Lie algebra of $Z_{G}(\mathfrak{a})$ (cf. [33, Proposition 24.3.6]). This completes the proof.

Let $H$ act on a complex algebraic variety $X$. A subgroup $\widetilde{H} \subseteq H$ is called a generic stabilizer for this action if there exists a non-empty open dense subset $U \subseteq X$ with the following property: the $H$-stabilizer of every $x \in U$ is conjugate to $\widetilde{H}$. A generic stabilizer is known to exist if $X$ is a linear representation of $H\left[32\right.$. We therefore have a generic stabilizer for the $H$-action on $\mathfrak{h}^{\perp}$, and we denote it by $H_{*}$. This group is known to be reductive (see [34, Theorem 9.1]).

Remark 16. A generic stabilizer is unique up to conjugation, meaning that $H_{*}$ more appropriately denotes a conjugacy class of subgroups in $H$. However, we shall always take $H_{*}$ to be a fixed subgroup in this conjugacy class.

Now recall our discussion of the the local structure theorem for a smooth, irreducible $G$-variety $Y$, as well as the notation introduced in that context (see 5.1). If $Y=G / H$, then the group $L_{0}$ turns out to be precisely $H_{*}$ (see [16, Section 8]).

Corollary 17. The pair $(G, H)$ is a-regular if and only if the connected component of the identity in $H_{*}$ is abelian.

Proof. Proposition [15 and the fact that $H_{*}=L_{0}$ reduce our task to one of proving that $Z_{G}(\mathfrak{a})=T$ if and only if the identity component in $L_{0}$ is abelian. To this end, consider [34, Definition 8.13] and [34, Proposition 8.14]. Since $G / H$ is an affine variety, these two statements imply that $L=Z_{G}(\mathfrak{a})$. Our task is therefore to prove that $L=T$ if and only if the identity component in $L_{0}$ is abelian. The forward implication follows immediately from the inclusion $L_{0} \subseteq L$, so that we only need to verify the opposite implication. 
Note that $L$ is a Levi factor of a parabolic subgroup of $G$, as discussed in 5.1 . This means that $L$ is connected and reductive, forcing the derived subgroup $[L, L]$ to be connected as well. The inclusion $[L, L] \subseteq L_{0}$ thus shows $[L, L]$ to be contained in the identity component in $L_{0}$. If we now assume that this component is abelian, then $[L, L]$ must also be abelian. It follows that $L$ is itself abelian. Together with the inclusion $T \subseteq L$ (see 5.1) and the fact that $L$ is a connected, reductive subgroup of $G$, this last sentence implies that $L=T$. The proof is complete.

Corollary 17 can be used to easily assess a-regularity in several examples. To see this, we note that [20] fully describes the $H$-representation $\mathfrak{h}^{\perp}$ in many cases. Each of these descriptions can be combined with the tables of Èlašvili [12,13] to compute $H_{*}$, after which Corollary 17 can be applied. We illustrate this in the following example.

Example 18. Consider the pair $(G, H)=\left(\mathrm{SL}_{p+q}, \mathrm{~S}\left(\mathrm{GL}_{p} \times \mathrm{GL}_{q}\right)\right)$ with $1 \leq p \leq q$. The vector space $\mathfrak{h}^{\perp}$ is isomorphic to $\left(\mathbb{C}^{p} \otimes\left(\mathbb{C}^{q}\right)^{*}\right) \oplus\left(\left(\mathbb{C}^{p}\right)^{*} \otimes \mathbb{C}^{q}\right)$ as an $H$-representation. The Lie algebra of the generic stabilizer for this action is isomorphic to $\mathbb{C}^{p} \oplus \mathfrak{s l}(q-p)$ if $p<q$ and to $\mathbb{C}^{p-1}$ if $p=q$. Hence $(G, H)$ is $\mathfrak{a}$-regular if and only if $q-p \leq 1$.

We now formulate a numerical criterion for a-regularity in terms of spherical-geometric invariants. Recall that the $\operatorname{rank} \operatorname{rk}_{G}(Y)$ of a $G$-variety $Y$ is the dimension of $\mathfrak{a}_{Y}$. The complexity $c_{G}(Y)$ of $Y$ is the codimension of a generic $B$-orbit in $Y$. We then have the following equalities, which are due to Knop [16]:

$$
\begin{aligned}
2 c_{G}(G / H)+\operatorname{rk}_{G}(G / H) & =\operatorname{dim} G-2 \operatorname{dim} H+\operatorname{dim} H_{*} \\
\operatorname{rk}_{G}(G / H) & =\operatorname{dim} T-\operatorname{dim} T_{*},
\end{aligned}
$$

where $T_{*}$ is a maximal torus of $H_{*}$.

Corollary 19. The pair $(G, H)$ is $\mathfrak{a}$-regular if and only if $c_{G}(G / H)+\operatorname{rk}_{G}(G / H)+\operatorname{dim} H=\operatorname{dim} B$.

Proof. Corollary 17 shows that $(G, H)$ is a-regular if and only if the identity component in $H_{*}$ is abelian. This is in turn equivalent to $\operatorname{dim} H_{*}=\operatorname{dim} T_{*}$, and the result then follows from (24) and (25).

The criteria established in Corollaries 17] and [19 become effective once we are able to either determine the Cartan space $\mathfrak{a}(\mathfrak{g}, \mathfrak{h})$ or the generic stabilizer $H_{*}$. The latter is difficult to accomplish in full generality, but Losev's work [25] makes the former achievable in a systematic way. Losev's method features prominently in the next subsection.

5.3. The Cartan space of a homogeneous affine variety. Continuing with the notation used in 5.2, we recall Losev's algorithm [25] for determining the Cartan space of $(G, H)$. We begin with the following definition (cf. [34, Section 10]).

Definition 20. The pair $(G, H)$ or the corresponding pair $(\mathfrak{g}, \mathfrak{h})$ is called:

(i) decomposable if there exist non-zero proper ideals $\mathfrak{g}_{1}, \mathfrak{g}_{2}$ in $\mathfrak{g}$ and any ideals $\mathfrak{h}_{1}, \mathfrak{h}_{2}$ in $\mathfrak{h}$ such that $\mathfrak{g}=\mathfrak{g}_{1} \oplus \mathfrak{g}_{2}, \mathfrak{h}=\mathfrak{h}_{1} \oplus \mathfrak{h}_{2}, \mathfrak{h}_{1} \subseteq \mathfrak{g}_{1}$, and $\mathfrak{h}_{2} \subseteq \mathfrak{g}_{2}$

(ii) indecomposable if it is not decomposable;

(iii) strictly indecomposable if $(\mathfrak{g},[\mathfrak{h}, \mathfrak{h}])$ is indecomposable.

We note that the Cartan space of a decomposable pair $\left(\mathfrak{g}_{1} \oplus \mathfrak{g}_{2}, \mathfrak{h}_{1} \oplus \mathfrak{h}_{2}\right)$ is $\mathfrak{a}\left(\mathfrak{g}_{1}, \mathfrak{h}_{1}\right) \oplus \mathfrak{a}\left(\mathfrak{g}_{2}, \mathfrak{h}_{2}\right)$. At the same time, observe that $\left(x_{1}, x_{2}\right) \in \mathfrak{g}_{1} \oplus \mathfrak{g}_{2}$ is a regular element if and only if $x_{1}$ and $x_{2}$ are regular elements of $\mathfrak{g}_{1}$ and $\mathfrak{g}_{2}$, respectively. These last two sentences imply that $\left(\mathfrak{g}_{1} \oplus \mathfrak{g}_{2}, \mathfrak{h}_{1} \oplus \mathfrak{h}_{2}\right)$ is $\mathfrak{a}$-regular if and only if $\left(\mathfrak{g}_{1}, \mathfrak{h}_{1}\right)$ and $\left(\mathfrak{g}_{2}, \mathfrak{h}_{2}\right)$ are $\mathfrak{a}$-regular. Recognizing its relevance to later arguments, we record this conclusion as follows. 
Lemma 21. Consider a collection of indecomposable pairs $\left(\mathfrak{g}_{i}, \mathfrak{h}_{i}\right), i=1, \ldots, n$, and suppose that our pair $(\mathfrak{g}, \mathfrak{h})$ is given by

$$
(\mathfrak{g}, \mathfrak{h})=\left(\bigoplus_{i=1}^{n} \mathfrak{g}_{i}, \bigoplus_{i=1}^{n} \mathfrak{h}_{i}\right)
$$

Then $(\mathfrak{g}, \mathfrak{h})$ is $\mathfrak{a}$-regular if and only if $\left(\mathfrak{g}_{i}, \mathfrak{h}_{i}\right)$ is $\mathfrak{a}$-regular for all $i=1, \ldots, n$.

Remark 22. Note that our pair $(\mathfrak{g}, \mathfrak{h})$ is necessarily expressible in the form (26), i.e. there exist indecomposable pairs $\left(\mathfrak{g}_{i}, \mathfrak{h}_{i}\right), i \in\{1, \ldots, n\}$, such that $\mathfrak{g}_{i}$ (resp. $\left.\mathfrak{h}_{i}\right)$ is an ideal in $\mathfrak{g}$ (resp. $\left.\mathfrak{h}\right)$ for all $i$ and (26) holds. This observation follows from Definition 20 via a straightforward induction argument, and it will be used implicitly in some of our arguments.

We now resume the main discussion. Note that for a subalgebra $\mathfrak{j} \subseteq \mathfrak{h}$, we have an inclusion $\mathfrak{a}(\mathfrak{g}, \mathfrak{h}) \subseteq \mathfrak{a}(\mathfrak{g}, \mathfrak{j})$ of Cartan spaces. It follows that $\mathfrak{a}(\mathfrak{g}, \mathfrak{h}) \subseteq \mathfrak{a}(\mathfrak{g}, \mathfrak{j})$ for all ideals $\mathfrak{j} \leq \mathfrak{h}$, which leads to the following definition (cf. [25, Definition 1.1]).

Definition 23. A reductive subalgebra $\mathfrak{j} \subseteq \mathfrak{g}$ is called essential if for every proper ideal $\mathfrak{i} \leq \mathfrak{j}$, the inclusion $\mathfrak{a}(\mathfrak{g}, \mathfrak{j}) \subseteq \mathfrak{a}(\mathfrak{g}, \mathfrak{i})$ is strict.

Now consider the Lie algebra $\mathfrak{h}_{*}$ of $H_{*}$, where $H_{*}$ is the generic stabilizer for the $H$-action on $\mathfrak{h}^{\perp}$ (see 5.2). Losev shows that $\mathfrak{h}_{*}$ generates an ideal $\mathfrak{h}_{\text {ess }} \leq \mathfrak{h}$ that is an essential subalgebra of $\mathfrak{g}$. This essential subalgebra is reductive and has the following properties:

- $\mathfrak{h}_{\text {ess }} \leq \mathfrak{h}$ is the unique ideal of $\mathfrak{h}$ for which $\mathfrak{a}(\mathfrak{g}, \mathfrak{h})=\mathfrak{a}\left(\mathfrak{g}, \mathfrak{h}_{\text {ess }}\right)$;

- $\mathfrak{h}_{\text {ess }}$ is maximal (for inclusion) among the ideals of $\mathfrak{h}$ that are essential subalgebras of $\mathfrak{g}$.

In principle, this reduces the computation of $\mathfrak{a}(\mathfrak{g}, \mathfrak{h})$ to the task of determining $\mathfrak{h}_{\text {ess }}$ and $\mathfrak{a}\left(\mathfrak{g}, \mathfrak{h}_{\text {ess }}\right)$.

The preceding discussion allows us to sketch the main results of [25]. Losev classifies the essential subalgebras $\mathfrak{j} \subseteq \mathfrak{g}$ that are semisimple, and in each such case he presents $\mathfrak{a}(\mathfrak{g}, \mathfrak{j})$ as the span of certain linear combinations of fundamental weights. This information may also be used to determine the Cartan space when $\mathfrak{j}$ is non-semisimple, provided that one knows the center of $\mathfrak{j}$. To this end, Losev gives an algorithm for calculating the centers of non-semisimple essential subalgebras.

5.4. Preliminaries for the classifications. We now discuss four items that are crucial to the classifications in 5.5. Our first item is the following elementary observation.

Observation 24. Let $\mathfrak{r}$ be a complex reductive Lie algebra with a reductive ideal $\mathfrak{i} \leq \mathfrak{r}$. If $\mathfrak{j}$ is a reductive ideal in $\mathfrak{i}$, then $\mathfrak{j}$ is also an ideal in $\mathfrak{r}$. This follows immediately from the decomposition of a reductive Lie algebra into a direct sum of its center and simple ideals, and it will be used implicitly in some of what follows.

We also need the following definition, which serves to formalize a standard idea.

Definition 25. Let $\mathfrak{r}_{1}$ and $\mathfrak{r}_{2}$ be complex Lie algebras with respective subalgebras $\mathfrak{s}_{1}$ and $\mathfrak{s}_{2}$. We refer to $\left(\mathfrak{r}_{1}, \mathfrak{s}_{1}\right)$ and $\left(\mathfrak{r}_{2}, \mathfrak{s}_{2}\right)$ as being conjugate if $\mathfrak{r}_{1}=\mathfrak{r}_{2}$ and $\mathfrak{s}_{1}=\phi\left(\mathfrak{s}_{2}\right)$ for some Lie algebra automorphism $\phi: \mathfrak{r}_{1} \rightarrow \mathfrak{r}_{1}$.

With this in mind, we have the following lemma.

Lemma 26. Assume that $\mathfrak{g}$ is simple and let $\mathfrak{h} \subseteq \mathfrak{g}$ be a reductive subalgebra.

(i) If $(\mathfrak{g}, \mathfrak{i})$ is not conjugate to a pair in Tables 1 or 2 from [25] for any ideal $\mathfrak{i} \leq \mathfrak{h}$, then $\mathfrak{h}_{\text {ess }}=\{0\}$. In this case, $\mathfrak{a}(\mathfrak{g}, \mathfrak{h})=\mathfrak{t}$ and $(\mathfrak{g}, \mathfrak{h})$ is $\mathfrak{a}$-regular.

(ii) If $\mathfrak{h}_{\mathrm{ess}} \neq\{0\}$, then $(\mathfrak{g}, \mathfrak{h})$ is $\mathfrak{a}$-regular if and only if $\left(\mathfrak{g},\left[\mathfrak{h}_{\mathrm{ess}}, \mathfrak{h}_{\mathrm{ess}}\right]\right)$ is conjugate to a pair in Table \ below. 


\begin{tabular}{c|c|c} 
& $\mathfrak{g}$ & $\mathfrak{i}$ \\
\hline 1 & $\mathfrak{s l}_{2 k}$ & $\mathfrak{s l}_{k} \oplus \mathfrak{s l}_{k}$ \\
2 & $\mathfrak{s l}_{2 k-1}$ & $\mathfrak{s l}_{k} \oplus \mathfrak{s l}_{k-1}$ \\
3 & $\mathfrak{s p}_{8}$ & $\mathfrak{s p}_{4} \oplus \mathfrak{s l}_{2} \oplus \mathfrak{s l}_{2}$ \\
4 & $\mathfrak{s p}_{6}$ & $\mathfrak{s l}_{2} \oplus \mathfrak{s l}_{2} \oplus \mathfrak{s l}_{2}$ \\
5 & $\mathfrak{e}_{6}$ & $\mathfrak{s l}_{6}$ \\
6 & $\mathfrak{s l}_{2 n+1}$ & $\mathfrak{s l}_{n+1}$ \\
7 & $\mathfrak{s l}_{2 n+1}$ & $\mathfrak{s p}_{2 n}$
\end{tabular}

TABLE I. For each line, the embedding $\mathfrak{i} \subseteq \mathfrak{g}$ is as described in [25, Section 6].

Proof. We begin by proving (i), and thus assume that $(\mathfrak{g}, \mathfrak{i})$ is not conjugate to a pair in Tables 1 or 2 from [25] for any ideal $\mathfrak{i} \leq \mathfrak{h}$. Noting the particular classification that each table gives, we conclude that $\left[\mathfrak{h}_{\text {ess }}, \mathfrak{h}_{\text {ess }}\right]$ cannot contain a non-zero semisimple ideal. Hence $\left[\mathfrak{h}_{\text {ess }}, \mathfrak{h}_{\text {ess }}\right]=\{0\}$, i.e. $\mathfrak{h}_{\text {ess }}$ is abelian. Since $\mathfrak{h}_{\text {ess }}$ is also reductive, one can find a Lie algebra automorphism of $\mathfrak{g}$ that sends $\mathfrak{h}_{\text {ess }}$ into $\mathfrak{t}$. This implies that $(\mathfrak{g}, \mathfrak{h})$ is conjugate to a pair $(\mathfrak{g}, \widetilde{\mathfrak{h}})$ satisfying $\widetilde{\mathfrak{h}}_{\text {ess }} \subseteq \mathfrak{t}$. We may therefore assume that $\mathfrak{h}_{\text {ess }} \subseteq \mathfrak{t}$.

Note that the inclusions $\{0\} \subseteq \mathfrak{h}_{\text {ess }} \subseteq \mathfrak{t}$ yield $\mathfrak{a}(\mathfrak{g}, \mathfrak{t}) \subseteq \mathfrak{a}\left(\mathfrak{g}, \mathfrak{h}_{\text {ess }}\right) \subseteq \mathfrak{a}(\mathfrak{g},\{0\})$, which by Example 12 amounts to the statement $\mathfrak{t} \subseteq \mathfrak{a}\left(\mathfrak{g}, \mathfrak{h}_{\text {ess }}\right) \subseteq \mathfrak{a}(\mathfrak{g},\{0\})$. At the same time, the inclusion $\mathfrak{a}(\mathfrak{g},\{0\}) \subseteq \mathfrak{t}$ follows from how we defined Cartan spaces in 5.1. We conclude that

$$
\mathfrak{t}=\mathfrak{a}\left(\mathfrak{g}, \mathfrak{h}_{\text {ess }}\right)=\mathfrak{a}(\mathfrak{g},\{0\}) .
$$

Recalling the properties of $\mathfrak{h}_{\text {ess }}$ discussed in 5.3, the first equality implies that $\mathfrak{t}=\mathfrak{a}(\mathfrak{g}, \mathfrak{h})$ and the second equality gives $\mathfrak{h}_{\text {ess }}=\{0\}$. The $\mathfrak{a}$-regularity of $(\mathfrak{g}, \mathfrak{h})$ now follows from the fact that $\mathfrak{t} \cap \mathfrak{g}_{\text {reg }} \neq \emptyset$, completing our proof of (i).

To prove (ii), we first assume that $\left(\mathfrak{g},\left[\mathfrak{h}_{\text {ess }}, \mathfrak{h}_{\text {ess }}\right]\right)$ is conjugate to a pair in Table【. If $\mathfrak{h}_{\text {ess }}$ is semisimple, i.e. $\left[\mathfrak{h}_{\text {ess }}, \mathfrak{h}_{\text {ess }}\right]=\mathfrak{h}_{\text {ess }}$, then $\mathfrak{a}\left(\mathfrak{g},\left[\mathfrak{h}_{\text {ess }}, \mathfrak{h}_{\text {ess }}\right]\right)=\mathfrak{a}\left(\mathfrak{g}, \mathfrak{h}_{\text {ess }}\right)=\mathfrak{a}(\mathfrak{g}, \mathfrak{h})$. This observation and an inspection of [25, Table 1] reveal that $(\mathfrak{g}, \mathfrak{h})$ is $\mathfrak{a}$-regular. If $\mathfrak{h}_{\text {ess }}$ is not semisimple, then $\left(\mathfrak{g},\left[\mathfrak{h}_{\text {ess }}, \mathfrak{h}_{\text {ess }}\right]\right)$ is conjugate to one of items 6 and 7 in Table $\mathbb{1}$ and $\mathfrak{h}_{\text {ess }}$ has a non-trivial center $\mathfrak{z}\left(\mathfrak{h}_{\text {ess }}\right)$. The result [25, Theorem 1.3(c)] then shows that

$$
\mathfrak{z}\left(\mathfrak{h}_{\mathrm{ess}}\right) \subseteq \mathfrak{z}:=\mathfrak{z}\left(\mathfrak{z}_{\mathfrak{g}}\left(\left[\mathfrak{h}_{\mathrm{ess}}, \mathfrak{h}_{\mathrm{ess}}\right]\right)\right)
$$

where $\mathfrak{z}_{\mathfrak{g}}\left(\left[\mathfrak{h}_{\text {ess }}, \mathfrak{h}_{\text {ess }}\right]\right)$ is the subalgebra of all elements in $\mathfrak{g}$ that commute with every element of $\left[\mathfrak{h}_{\text {ess }}, \mathfrak{h}_{\text {ess }}\right]$ and $\mathfrak{z}\left(\mathfrak{z}_{\mathfrak{g}}\left(\left[\mathfrak{h}_{\text {ess }}, \mathfrak{h}_{\text {ess }}\right]\right)\right)$ is the center of this subalgebra. Noting again that $\left(\mathfrak{g},\left[\mathfrak{h}_{\text {ess }}, \mathfrak{h}_{\text {ess }}\right]\right)$ is conjugate to item 6 or 7 in Table I] one uses [25, Table 2] to see that $\mathfrak{a}\left(\mathfrak{g},\left[\mathfrak{h}_{\text {ess }}, \mathfrak{h}_{\text {ess }}\right]+\mathfrak{z}\right)$ has regular elements. Note also that

implies

$$
\mathfrak{h}_{\text {ess }}=\left[\mathfrak{h}_{\text {ess }}, \mathfrak{h}_{\text {ess }}\right]+\mathfrak{z}\left(\mathfrak{h}_{\text {ess }}\right) \subseteq\left[\mathfrak{h}_{\text {ess }}, \mathfrak{h}_{\text {ess }}\right]+\mathfrak{z}
$$

$$
\mathfrak{a}\left(\mathfrak{g},\left[\mathfrak{h}_{\text {ess }}, \mathfrak{h}_{\text {ess }}\right]+\mathfrak{z}\right) \subseteq \mathfrak{a}\left(\mathfrak{g}, \mathfrak{h}_{\text {ess }}\right)=\mathfrak{a}(\mathfrak{g}, \mathfrak{h}) .
$$

The previous two sentences together show that $(\mathfrak{g}, \mathfrak{h})$ is $\mathfrak{a}$-regular.

For the converse we suppose that $\mathfrak{h}_{\text {ess }} \leq \mathfrak{h}$ is not the trivial ideal. The discussion above implies that $\mathfrak{h}_{\text {ess }}$ cannot be abelian, so that $\left[\mathfrak{h}_{\text {ess }}, \mathfrak{h}_{\text {ess }}\right] \leq \mathfrak{h}$ is a semisimple and non-trivial ideal. It then follows from Losev's setup in [25] that $\left[\mathfrak{h}_{\text {ess }}, \mathfrak{h}_{\text {ess }}\right]$ is conjugate to a pair in [25, Table 1] or [25, Table $2]$. Hence there are three mutually exclusive possibilities: $\left(\mathfrak{g},\left[\mathfrak{h}_{\mathrm{ess}}, \mathfrak{h}_{\mathrm{ess}}\right]\right)$ is conjugate to a pair in:

(a) [25, Table 1], but not to one in [25, Table 2];

(b) [25, Table 1] and [25, Table 2];

(c) [25, Table 2], but not to one in [25, Table 1]. 
In each instance, we simply use Losev's tables to inspect all possible Cartan spaces $\mathfrak{a}(\mathfrak{g}, \mathfrak{h})$ and determine whether each has a regular element.

We first suppose that (a) holds. Then $(\mathfrak{g}, \mathfrak{h})$ is $\mathfrak{a}$-regular precisely when $(\mathfrak{g}, \mathfrak{h}$ ess $)$ is conjugate to one of the items 2 (with $k=n / 2,(n+1) / 2$ ), 6 (with $n=4$ ), 7 or 21 from [25, Table 1]. These pairs constitute the first five lines of Table I.

Now suppose that (b) holds. Then $\left(\mathfrak{g},\left[\mathfrak{h}_{\text {ess }}, \mathfrak{h}_{\text {ess }}\right]\right)$ is conjugate to one of the items 1,2 (with $n / 2<$ $k \leq n-2)$, 10 or 19 from [25, Table 1]. A case-by-case examination reveals that $\left(\mathfrak{g},\left[\mathfrak{h}_{\text {ess }}, \mathfrak{h}_{\text {ess }}\right]\right)$ is not $\mathfrak{a}$-regular, i.e. $\mathfrak{a}\left(\mathfrak{g},\left[\mathfrak{h}_{\text {ess }}, \mathfrak{h}_{\text {ess }}\right]\right) \cap \mathfrak{g}_{\text {reg }}=\emptyset$. It then follows from the inclusion $\mathfrak{a}\left(\mathfrak{g}, \mathfrak{h}_{\text {ess }}\right) \subseteq \mathfrak{a}\left(\mathfrak{g},\left[\mathfrak{h}_{\text {ess }}, \mathfrak{h}_{\text {ess }}\right]\right)$ that $\mathfrak{a}\left(\mathfrak{g}, \mathfrak{h}_{\text {ess }}\right) \cap \mathfrak{g}_{\text {reg }}=\emptyset$. Since $\mathfrak{a}(\mathfrak{g}, \mathfrak{h}$ ess $)=\mathfrak{a}(\mathfrak{g}, \mathfrak{h})$, this means that $(\mathfrak{g}, \mathfrak{h})$ is not $\mathfrak{a}$-regular.

We last suppose that (c) holds, in which case $\left(\mathfrak{g},\left[\mathfrak{h}_{\text {ess }}, \mathfrak{h}_{\text {ess }}\right]\right)$ is conjugate to item 6 or 7 in Table $\mathbb{I}$ As argued above, the pair $(\mathfrak{g}, \mathfrak{h})$ is necessarily $\mathfrak{a}$-regular.

For the last preliminary topic, let $H$ be any reductive subgroup of our connected semisimple group $G$. The coordinate ring $\mathbb{C}[G / H]$ then decomposes into certain irreducible, highest-weight $G$-modules, and the highest weights appearing in this decomposition are the so-called spherical weights. These weights form a finitely generated semigroup $\Lambda_{+}(G, H)$. With this in mind, we record the following immediate consequence of [34, Proposition 5.14].

Lemma 27. If $H$ is any closed, reductive subgroup of $G$, then $\mathfrak{a}(\mathfrak{g}, \mathfrak{h})^{*}$ is spanned by $\Lambda_{+}(G, H)$.

5.5. The classifications. We maintain the notation used in 5.3, and now address the classification of $\mathfrak{a}$-regular pairs $(G, H)$ (equivalently, a-regular pairs $(\mathfrak{g}, \mathfrak{h}))$ in each of the following three cases: $H$ is a Levi subgroup 5.5.1, $H$ is symmetric 5.5.2, and $H$ is simultaneously reductive, spherical, and nonsymmetric 5.5.3. In each case, we reduce to the classification of strictly indecomposable, a-regular pairs. We list all conjugacy classes of such pairs in each of the cases 5.5.2 and 5.5.3, where the notion of conjugacy class comes from Definition 25.

Remark 28. We emphasize that the classification of strictly indecomposable pairs works differently in each of the above-mentioned cases. In the case of Levi subgroups $H \subseteq G$, the classification is almost entirely based on Losev's work [25. This is in contrast to the case of symmetric subgroups, in which we appeal to representation-theoretic results about symmetric spaces. Several of these results are not applicable to the case of a reductive spherical $H \subseteq G$, for which we instead harness the works of Brion [7], Krämer [21], and Mikityuk [28].

Remark 29. Note that every symmetric subgroup of $G$ is reductive and spherical (see [34, Theorem 26.14]). The techniques and arguments in 5.5.3 thereby imply the classification results in 5.5.2. Despite this, we believe that the representation-theoretic approach taken in 5.5.2 is independently interesting and worthwhile. Further distinctions between 5.5.2 and 5.5.3 are discussed in Remark 32 and Example 36.

5.5.1. Levi subgroups. Assume that $H$ is a Levi subgroup of $G$, by which we mean that $H$ is a Levi factor of a parabolic subgroup $P \subseteq G$. It follows that $\mathfrak{h}$ is a Levi factor of a parabolic subalgebra $\mathfrak{p} \subseteq \mathfrak{g}$. Now let $\mathfrak{g}=\mathfrak{g}_{1} \oplus \cdots \oplus \mathfrak{g}_{n}$ be the decomposition of $\mathfrak{g}$ into its simple ideals $\mathfrak{g}_{1}, \ldots, \mathfrak{g}_{n}$. The parabolic subalgebra $\mathfrak{p}$ is then a sum of parabolic subalgebras $\mathfrak{p}_{i} \subseteq \mathfrak{g}_{i}$ for $i=1, \ldots, n$, implying that $\mathfrak{h}$ is a sum of Levi factors $\mathfrak{h}_{i} \subseteq \mathfrak{p}_{i}, i=1, \ldots, n$. An application of Lemma 21 then shows that $(\mathfrak{g}, \mathfrak{h})$ is $\mathfrak{a}$-regular if and only if $\left(\mathfrak{g}_{i}, \mathfrak{h}_{i}\right)$ is $\mathfrak{a}$-regular for all $i=1, \ldots, n$. It therefore suffices to assume that $\mathfrak{g}$ is simple. Our classification then takes the following form.

Proposition 30. Assume that $\mathfrak{g}$ is simple and that $\mathfrak{h}$ is a Levi subalgebra of $\mathfrak{g}$ with $\mathfrak{h}_{\mathrm{ess}} \neq\{0\}$. The pair $(\mathfrak{g}, \mathfrak{h})$ is then $\mathfrak{a}$-regular if and only if it is conjugate to a pair in Table II. In this table, $\mathfrak{l}_{2}$ is any reductive subalgebra of $\mathfrak{s l}_{2 n+1}$ that satisfies the following conditions: $\mathfrak{s l}_{n+1} \cap \mathfrak{l}_{2}=\{0\}, \mathfrak{l}_{2}$ commutes with $\mathfrak{s l}_{n+1}$, and $\mathfrak{s l}_{n+1} \oplus \mathfrak{l}_{2}$ is a Levi subalgebra of $\mathfrak{s l}_{2 n+1}$ 1

\footnotetext{
${ }^{1}$ We are implicitly using the embedding $\mathfrak{s l}_{n+1} \subseteq \mathfrak{s l}_{2 n+1}$ from line 6 of Table $\square$
} 


\begin{tabular}{c|c|c} 
& $\mathfrak{g}$ & $\mathfrak{l}$ \\
\hline 1 & $\mathfrak{s l}_{2 k}$ & $\mathfrak{s}\left(\mathfrak{g l}_{k} \oplus \mathfrak{g l}_{k}\right)$ \\
2 & $\mathfrak{s l}_{2 k-1}$ & $\mathfrak{s}\left(\mathfrak{g l}_{k} \oplus \mathfrak{g l}_{k-1}\right)$ \\
3 & $\mathfrak{e}_{6}$ & $\mathfrak{s l}_{6} \oplus \mathbb{C}$ \\
4 & $\mathfrak{s l}_{2 n+1}$ & $\mathfrak{s l}_{n+1} \oplus \mathfrak{l}_{2}$
\end{tabular}

TABLE II. Line 3 is to be understood as follows. Up to Lie algebra automorphism, $\mathfrak{e}_{6}$ contains precisely one subalgebra isomorphic to $\mathfrak{s l}_{6} \oplus \mathfrak{s l}_{2}$ (see [11, Theorem 5.5, Table 12, and Theorem 11.1]). By choosing a Cartan subalgebra of $\mathfrak{s l}_{2}$ and identifying it with $\mathbb{C}$, one obtains a unique automorphism class of subalgebras in $\mathfrak{e}_{6}$ that are isomorphic to $\mathfrak{s l}_{6} \oplus \mathbb{C}$. This turns out to be a class of Levi subalgebras in $\mathfrak{e}_{6}$, and the reader may take any of these to be the subalgebra $\mathfrak{l}$ in line 3 .

Proof. We first assume that $(\mathfrak{g}, \mathfrak{h})$ is conjugate to a pair in Table II A case-by-case analysis reveals that each pair in Table $\amalg$ is $\mathfrak{a}$-regular, implying that $(\mathfrak{g}, \mathfrak{h})$ is $\mathfrak{a}$-regular.

Conversely, assume that $(\mathfrak{g}, \mathfrak{h})$ is $\mathfrak{a}$-regular. Lemma 26)(ii) then implies the existence of an ideal $\mathfrak{i}$ in $\mathfrak{h}$ for which $(\mathfrak{g}, \mathfrak{i})$ is conjugate to a pair in Table I. We will therefore begin by finding the pairs in Table 【for which this is possible. For each such pair $(\mathfrak{r}, \mathfrak{j})$, we will subsequently find the Levi subalgebras $\mathfrak{l} \subseteq \mathfrak{r}$ that contain $\mathfrak{j}$ as an ideal. Note that $(\mathfrak{g}, \mathfrak{h})$ will then be conjugate to one of the pairs $(\mathfrak{r}, \mathfrak{l})$ arising in this way. It will then suffice to observe that the aforementioned pairs $(\mathfrak{r}, \mathfrak{l})$ appear in Table II

Let $(\mathfrak{r}, \mathfrak{j})$ be any of the pairs appearing in lines 3,4, and 7 of Table I. Observe that the Dynkin diagram of $\mathfrak{j}$ is not a subdiagram in the Dynkin diagram of $\mathfrak{r}$. At the same time, the Dynkin diagram of any ideal in a Levi subalgebra of $\mathfrak{g}$ must be a subdiagram in the Dynkin diagram of $\mathfrak{g}$. It follows that $(\mathfrak{g}, \mathfrak{i})$ cannot be conjugate to $(\mathfrak{r}, \mathfrak{j})$ for any ideal $\mathfrak{i} \leq \mathfrak{h}$.

In light of the previous paragraph, we may restrict our attention to the pairs in lines $1,2,5$, and 6 of Table I. Let $(\mathfrak{r}, \mathfrak{j})$ be any such pair, recalling that the embedding of $\mathfrak{j}$ into $\mathfrak{r}$ is described in [25, Section 6] (cf. the caption of Table I). This description is easily seen to imply that $j$ is an ideal in a Levi subalgebra of $\mathfrak{r}$. If $(\mathfrak{r}, \mathfrak{j})$ is in one of lines 1,2 , and 5 from Table प then the Dynkin diagram of $\mathfrak{j}$ uniquely determines a Levi subalgebra $\mathfrak{l} \subseteq \mathfrak{r}$ that contains $\mathfrak{j}$ as an ideal. The pair $(\mathfrak{r}, \mathfrak{l})$ is recorded in Table II. If $(\mathfrak{r}, \mathfrak{j})$ is in line 6 from Table I. i.e. $\mathfrak{r}=\mathfrak{s l}_{2 n+1}$ and $\mathfrak{j}=\mathfrak{s l}_{n+1}$, then there are several Levi subalgebras $\mathfrak{l} \subseteq \mathfrak{r}$ that contain $\mathfrak{j}$ as an ideal. The Dynkin diagram of any such $\mathfrak{l}$ is a subdiagram in the Dynkin diagram of $\mathfrak{s l}_{2 n+1}$, and it contains the Dynkin diagram of $\mathfrak{s l}_{n+1}$ as a connected component. It follows that $\mathfrak{l}=\mathfrak{s l}_{n+1} \oplus \mathfrak{l}_{2}$ for some reductive subalgebra $\mathfrak{l}_{2} \subseteq \mathfrak{s l}_{2 n+1}$ that satisfies the desired hypotheses.

5.5.2. Symmetric subgroups. Using the notation established in 5.1 and the introduction of Section 3. we assume that the subgroup $H \subseteq G$ is symmetric. This means that $H$ is an open subgroup of $G^{\theta}$, the subgroup of fixed points of an involutive algebraic group automorphism $\theta: G \rightarrow G$. It follows that $(\mathfrak{g}, \mathfrak{h})$ is a symmetric pair, i.e. $\mathfrak{h}$ coincides with the set of $\theta$-fixed vectors $\mathfrak{g}^{\theta} \subseteq \mathfrak{g}$ for the corresponding involutive Lie algebra automorphism $\theta: \mathfrak{g} \rightarrow \mathfrak{g}$.

Lemma 31. If $\mathfrak{h}$ is any reductive subalgebra of $\mathfrak{g}$, then $(\mathfrak{g}, \mathfrak{h})$ is a symmetric pair if and only if there exist strictly indecomposable symmetric pairs $\left(\mathfrak{g}_{i}, \mathfrak{h}_{i}\right), i \in\{1, \ldots, n\}$, such that $\mathfrak{g}_{i}$ (resp. $\mathfrak{h}_{i}$ ) is an ideal in $\mathfrak{g}$ (resp. $\mathfrak{h})$ for all $i$ and

$$
(\mathfrak{g}, \mathfrak{h})=\left(\bigoplus_{i=1}^{n} \mathfrak{g}_{i}, \bigoplus_{i=1}^{n} \mathfrak{h}_{i}\right)
$$


Proof. The backward implication follows from the following simple observation: if $\left(\mathfrak{g}_{1}, \mathfrak{h}_{1}\right)$ and $\left(\mathfrak{g}_{2}, \mathfrak{h}_{2}\right)$ are symmetric pairs, then $\left(\mathfrak{g}_{1} \oplus \mathfrak{g}_{2}, \mathfrak{h}_{1} \oplus \mathfrak{h}_{2}\right)$ is also a symmetric pair.

To prove the forward implication, assume that $(\mathfrak{g}, \mathfrak{h})$ is a symmetric pair and let $\theta: \mathfrak{g} \rightarrow \mathfrak{g}$ be an involutive automorphism for which $\mathfrak{h}=\mathfrak{g}^{\theta}$. Note that each simple ideal of $\mathfrak{g}$ is either $\theta$-stable or interchanged by $\theta$ with a different simple ideal. We may therefore identify $\mathfrak{g}$ with

$$
\mathfrak{g}_{1}^{\oplus 2} \cdots \oplus \mathfrak{g}_{s}^{\oplus 2} \oplus \mathfrak{g}_{s+1} \cdots \oplus \mathfrak{g}_{s+t}
$$

for simple Lie algebras $\mathfrak{g}_{1}, \ldots, \mathfrak{g}_{s+t}$, such that $\theta$ becomes the following map: $(x, y) \mapsto(y, x)$ on each summand $\mathfrak{g}_{i}^{\oplus 2}$ and $x \mapsto \theta_{j}(x)$ on each summand $\mathfrak{g}_{j}$, where $\theta_{j}: \mathfrak{g}_{j} \mapsto \mathfrak{g}_{j}$ is an involutive automorphism. It follows that

$$
\mathfrak{h}=\mathfrak{g}^{\theta}=\operatorname{diag}\left(\mathfrak{g}_{1}\right) \oplus \cdots \oplus \operatorname{diag}\left(\mathfrak{g}_{s}\right) \oplus \mathfrak{g}_{s+1}^{\theta_{s+1}} \oplus \cdots \oplus \mathfrak{g}_{s+t}^{\theta_{s+t}},
$$

where $\operatorname{diag}\left(\mathfrak{g}_{i}\right):=\left\{(x, y) \in \mathfrak{g}_{i}^{\oplus 2}: x=y\right\}$ for all $i \in\{1, \ldots, s\}$.

In light of the above, it suffices to prove that the symmetric pairs $\left(\mathfrak{g}_{i}^{\oplus 2}, \operatorname{diag}\left(\mathfrak{g}_{i}\right)\right)$ and $\left(\mathfrak{g}_{j}, \mathfrak{g}_{j}^{\theta_{j}}\right)$ are strictly indecomposable for all $i \in\{1, \ldots, s\}$ and $j \in\{s+1, \ldots, s+t\}$. The strict indecomposability of the latter pair follows from the fact that $\mathfrak{g}_{j}$ is simple. Now observe that the simplicity of $\mathfrak{g}_{i} \cong$ $\operatorname{diag}\left(\mathfrak{g}_{i}\right)$ implies that $\left(\mathfrak{g}_{i}^{\oplus 2},\left[\operatorname{diag}\left(\mathfrak{g}_{i}\right), \operatorname{diag}\left(\mathfrak{g}_{i}\right)\right]\right)=\left(\mathfrak{g}_{i}^{\oplus 2}, \operatorname{diag}\left(\mathfrak{g}_{i}\right)\right)$. It follows that $\left(\mathfrak{g}_{i}^{\oplus 2}, \operatorname{diag}\left(\mathfrak{g}_{i}\right)\right)$ is strictly indecomposable if and only if it is indecomposable. However, $\operatorname{since} \operatorname{diag}\left(\mathfrak{g}_{i}\right)$ is simple, the decomposability of $\left(\mathfrak{g}_{i}^{\oplus 2}, \operatorname{diag}\left(\mathfrak{g}_{i}\right)\right)$ would entail $\operatorname{diag}\left(\mathfrak{g}_{i}\right)$ being contained in a proper ideal of $\mathfrak{g}_{i}^{\oplus 2}$. This is not possible, meaning that $\left(\mathfrak{g}_{i}^{\oplus 2}, \operatorname{diag}\left(\mathfrak{g}_{i}\right)\right)$ is indeed strictly indecomposable. The proof is complete.

Remark 32. One immediate consequence is that every indecomposable symmetric pair $(\mathfrak{g}, \mathfrak{h})$ is strictly indecomposable. This is not true of an arbitrary reductive spherical pair $(\mathfrak{g}, \mathfrak{h})$ (see Example 36).

Together with Lemma 21, Lemma 31 reduces the classification of a-regular symmetric pairs to the classification of $\mathfrak{a}$-regular, strictly indecomposable symmetric pairs. Let $(\mathfrak{g}, \mathfrak{h})$ be a pair of the latter sort, and let $(G, H)$ denote an associated pair of groups. Let us also consider an involutive automorphism $\theta: \mathfrak{g} \rightarrow \mathfrak{g}$ satisfying $\mathfrak{h}=\mathfrak{g}^{\theta}$. This forms part of the eigenspace decomposition $\mathfrak{g}=\mathfrak{h} \oplus \mathfrak{q}$, where $\mathfrak{q} \subseteq \mathfrak{g}$ is the -1 -eigenspace of $\theta$. One can then find a maximal abelian subspace $\mathfrak{c} \subseteq \mathfrak{q}$, meaning that $\mathfrak{c}$ is a vector subspace of $\mathfrak{q}$ that is maximal with respect to the following condition: $\mathfrak{c}$ is abelian and consists of semisimple elements in $\mathfrak{g}$ (cf. [33, Corollary 37.5.4]).

Now recall our discussion of the generic stabilizer $H_{*} \subseteq H$ and its Lie algebra $\mathfrak{h}_{*} \subseteq \mathfrak{h}$ (see 5.2 and 5.3). At the same time, let $\mathfrak{z}_{\mathfrak{h}}(Y)$ denote the subalgebra of all $x \in \mathfrak{h}$ that commute with every vector in a subset $Y \subseteq \mathfrak{g}$.

Lemma 33. We have $\mathfrak{h}_{*}=\mathfrak{z}_{\mathfrak{h}}(\mathfrak{c})$.

Proof. The $H$-module isomorphisms $\mathfrak{h}^{\perp} \cong \mathfrak{g} / \mathfrak{h} \cong \mathfrak{q}$ imply that $H_{*}$ is a generic stabilizer for the $H$ action on $\mathfrak{q}$. Note also that $H \cdot \mathfrak{c} \subseteq \mathfrak{q}$ is dense (see [33, Lemma 38.7.1]) and constructible. It follows that $\mathfrak{h}_{*}=\mathfrak{z}_{\mathfrak{h}}(c)$ for all $c$ in an open dense subset $\mathfrak{c}_{1} \subseteq \mathfrak{c}$. At the same time, there exists an open dense subset $\mathfrak{c}_{2} \subseteq \mathfrak{c}$ with the property that $\mathfrak{z}_{\mathfrak{h}}(c)=\mathfrak{z}_{\mathfrak{h}}(\mathfrak{c})$ for all $c \in \mathfrak{c}_{2}$ (see the proof of [33, Proposition 38.4.5]). Hence $\mathfrak{h}_{*}=\mathfrak{z}_{\mathfrak{h}}(\mathfrak{c})$, as desired.

Remark 34. With Remark 16 in mind, one can phrase Lemma 33 as follows: $\mathfrak{z h}_{\mathfrak{h}}(\mathfrak{c})$ represents the conjugacy class of Lie algebras of generic stabilizers for the $H$-action on $\mathfrak{h}^{\perp}$.

We now explain the classification of $\mathfrak{a}$-regular, strictly indecomposable symmetric pairs $(\mathfrak{g}, \mathfrak{h})$. Up to conjugation (see Definition 25), such pairs are parametrized by Satake diagrams (see [34, Section $26.5])$. The Satake diagram for a symmetric pair $(\mathfrak{g}, \mathfrak{h})$ is the Dynkin diagram of $\mathfrak{g}$, together with extra decorations that encode the associated involution $\theta: \mathfrak{g} \rightarrow \mathfrak{g}$. Part of this decoration consists 
of painting some of the nodes black; these are precisely the simple roots of $\mathfrak{z}_{\mathfrak{h}}(\mathfrak{c})$. At the same time, recall that Lemma 33 identifies $\mathfrak{z}_{\mathfrak{h}}(\mathfrak{c})$ with $\mathfrak{h}_{*}$. Appealing to Corollary [17, we see that the $\mathfrak{a}$-regularity of $(\mathfrak{g}, \mathfrak{h})$ is equivalent to the Satake diagram of $(\mathfrak{g}, \mathfrak{h})$ having none of its nodes painted black. This leads to the following result.

Proposition 35. A strictly indecomposable symmetric pair $(\mathfrak{g}, \mathfrak{h})$ is $\mathfrak{a}$-regular if and only if it is conjugate to one of the pairs in Table III. In this table, $\mathfrak{s}$ denotes any simple Lie algebra.

\begin{tabular}{c|c|c} 
& $\mathfrak{g}$ & $\mathfrak{h}$ \\
\hline 1 & $\mathfrak{s l}_{n}$ & $\mathfrak{s o}_{n}$ \\
\hline 2 & $\mathfrak{s l}_{2 n+1}$ & $\mathfrak{s l}_{n+1} \oplus \mathfrak{s l}_{n} \oplus \mathbb{C}$ \\
& $\mathfrak{s l}_{2 n}$ & $\mathfrak{s l}_{n} \oplus \mathfrak{s l}_{n} \oplus \mathbb{C}$ \\
\hline 3 & $\mathfrak{s o}_{2 n+1}$ & $\mathfrak{s o}_{n+1} \oplus \mathfrak{s o}_{n}$ \\
& $\mathfrak{s o}_{2 n}$ & $\mathfrak{s o}_{n} \oplus \mathfrak{s o}_{n}$ \\
& $\mathfrak{s o}_{2 n}$ & $\mathfrak{s o}_{n-1} \oplus \mathfrak{s o}_{n+1}$ \\
\hline 4 & $\mathfrak{s p}_{2 n}$ & $\mathfrak{g l}_{n}$ \\
\hline 5 & $\mathfrak{e}_{6}$ & $\mathfrak{s p}_{8}$ \\
\hline 6 & $\mathfrak{e}_{6}$ & $\mathfrak{s l}_{6} \oplus \mathfrak{s l}_{2}$ \\
\hline 7 & $\mathfrak{e}_{7}$ & $\mathfrak{s l}_{8}$ \\
\hline 8 & $\mathfrak{e}_{8}$ & $\mathfrak{s o}_{16}$ \\
\hline 9 & $\mathfrak{f}_{4}$ & $\mathfrak{s p}_{6} \oplus \mathfrak{s l}_{2}$ \\
\hline 10 & $\mathfrak{g}_{2}$ & $\mathfrak{s l}_{2} \oplus \mathfrak{s l}_{2}$ \\
\hline 11 & $\mathfrak{s} \oplus \mathfrak{s}$ & $\operatorname{diag}(\mathfrak{s})$
\end{tabular}

TABLE III. The embeddings $\mathfrak{h} \subseteq \mathfrak{g}$ are obtained from [21, Table 1], which describes each embedding on the level of algebraic groups.

Proof. Following the discussion above, we only need to list the symmetric pairs whose Satake diagrams have no black nodes. These diagrams can be found in [34, Table 26.3], and the result follows from an inspection of this table.

5.5.3. Reductive spherical subgroups. Using the notation in 5.1 and the introduction of Section 3 , we additionally assume that $(G, H)$ and $(\mathfrak{g}, \mathfrak{h})$ are reductive spherical pairs. This means that $H$ is a reductive spherical subgroup of $G$, i.e. $H$ is reductive and $B$ has an open orbit in $G / H$. Note that this is equivalent to $\mathfrak{h}$ being a reductive subalgebra of $\mathfrak{g}$ satisfying $\widetilde{\mathfrak{b}}+\mathfrak{h}=\mathfrak{g}$ for some Borel subalgebra $\widetilde{\mathfrak{b}} \subseteq \mathfrak{g}$ (see [34, Section 25.1]). We shall sometimes also require $(G, H)$ and $(\mathfrak{g}, \mathfrak{h})$ to be non-symmetric, noting that the classification in 5.5 .2 renders this a harmless assumption.

Example 36. In contrast to the situation considered in Remark 32, an indecomposable reductive spherical pair need not be strictly indecomposable. Set $\mathfrak{g}=\mathfrak{s l}_{n+1} \oplus \mathfrak{s l}_{2}$ and let $\mathfrak{h} \subseteq \mathfrak{g}$ be the image of

$$
\mathfrak{s l}_{n} \oplus \mathbb{C} \rightarrow \mathfrak{g}, \quad(A, t) \mapsto\left(\operatorname{diag}\left(A+t I_{n},-n t\right), \operatorname{diag}(t,-t)\right), \quad(A, t) \in \mathfrak{s l}_{n} \oplus \mathbb{C} .
$$

This is an indecomposable spherical pair, but it is not strictly indecomposable.

Remark 37. The strictly indecomposable reductive spherical pairs $(G, H)$ have been classified by Krämer [21] for $G$ simple, and by Mikityuk [28] and Brion [7] for $G$ semisimple. 
We begin by assuming that our reductive spherical pair $(G, H)$ is strictly indecomposable. Now note that Lemma 27 allows us to investigate a-regularity via $\Lambda_{+}(G, H)$, and the case-by-case analyses of [21] thereby become important. The aforementioned reference gives explicit semigroup generators of $\Lambda_{+}(G, H)$ if $G$ is simple. If $G$ is only semisimple, then a description of $\Lambda_{+}(G, H)$ can be obtained from [3, Table 1] as follows. If $\mathfrak{h}$ has a trivial center, then generators of $\Lambda_{+}(G, H)$ are given in [3, Table 1]. If $\mathfrak{h}$ has a non-trivial center, then [3, Table 1] provides a finite set $\left\{\left(\lambda_{1}, \chi_{1}\right), \ldots,\left(\lambda_{s}, \chi_{s}\right)\right\}$ of generators for the so-called extended weight semigroup of $(G, H)$. The $\lambda_{i}$ are dominant weights for $G$ and the $\chi_{i}$ are characters of $H$. The weight semigroup $\Lambda_{+}(G, H)$ identifies with the collection of all points in the extended weight semigroup that have the form $(\lambda, 0)$. This amounts to the following statement: a dominant weight $\lambda$ belongs to $\Lambda_{+}(G, H)$ if and only if $\lambda=\sum_{i=1}^{s} n_{i} \lambda_{i}$ for some non-negative integers $n_{i}$ satisfying $\sum_{i=1}^{s} n_{i} \chi_{i}=0$. Together with an inspection of [21, Table 1] and [3, Table 1], this discussion yields the following fact.

Lemma 38. If $(G, H)$ is a strictly indecomposable reductive spherical pair that is not symmetric, then $(G, H)$ is $\mathfrak{a}$-regular if and only if $(\mathfrak{g}, \mathfrak{h})$ is conjugate to a pair in Table IV.

\begin{tabular}{|c|c|c|}
\hline & $\mathfrak{g}$ & $\mathfrak{h}$ \\
\hline 1 & $\mathfrak{s l}_{2 n+1}$ & $\mathfrak{s l}_{n+1} \oplus \mathfrak{s l}_{n}$ \\
\hline 2 & $\mathfrak{s l}_{2 n+1}$ & $\mathfrak{s p}_{2 n} \oplus \mathbb{C}$ \\
\hline 3 & $\mathfrak{s l}_{2 n+1}$ & $\mathfrak{s p}_{2 n}$ \\
\hline 4 & $\mathfrak{s o}_{2 n+1}$ & $\mathfrak{g l}_{n}$ \\
\hline 5 & $\mathfrak{s l}_{n+1} \oplus \mathfrak{s l}_{n}$ & $\mathfrak{s l}_{n} \oplus \mathbb{C}$ \\
\hline 6 & $\mathfrak{s o}_{n+1} \oplus \mathfrak{s o}_{n}$ & $\mathfrak{s o}_{n}$ \\
\hline 7 & $\begin{array}{c}\mathfrak{s l}_{n} \oplus \mathfrak{s p}_{2 m} \\
(n=3,4,5, m=1,2)\end{array}$ & $\mathfrak{g l}_{n-2} \oplus \mathfrak{s l}_{2} \oplus \mathfrak{s p}_{2 m-2}$ \\
\hline 8 & $\begin{array}{c}\mathfrak{s l}_{n} \oplus \mathfrak{s p}_{2 m} \\
(n=3,5, m=1,2)\end{array}$ & $\mathfrak{s l}_{n-2} \oplus \mathfrak{s l}_{2} \oplus \mathfrak{s p}_{2 m-2}$ \\
\hline 9 & $\begin{array}{l}\mathfrak{s p}_{2 m} \oplus \mathfrak{s p}_{2 n} \\
(m, n=1,2)\end{array}$ & $\mathfrak{s p}_{2 n-2} \oplus \mathfrak{s p}_{2} \oplus \mathfrak{s p}_{2 m-2}$ \\
\hline 10 & $\begin{array}{c}\mathfrak{s p}_{2 n} \oplus \mathfrak{s p}_{4} \\
(n=3,4)\end{array}$ & $\mathfrak{s p}_{2 n-4} \oplus \mathfrak{s p}_{4}$ \\
\hline 11 & $\begin{array}{c}\mathfrak{s p}_{2 \ell} \oplus \mathfrak{s p}_{2 m} \oplus \mathfrak{s p}_{2 n} \\
(\ell, m, n=1,2)\end{array}$ & $\mathfrak{s l}_{2 \ell-2} \oplus \mathfrak{s p}_{2 m-2} \oplus \mathfrak{s p}_{2 n-2} \oplus \mathfrak{s p}_{2}$ \\
\hline 12 & $\begin{array}{c}\mathfrak{s p}_{2 n} \oplus \mathfrak{s p}_{4} \oplus \mathfrak{s p}_{2 m} \\
(n, m=1,2)\end{array}$ & $\mathfrak{s p}_{2 n-2} \oplus \mathfrak{s p}_{2} \oplus \mathfrak{s p}_{2} \oplus \mathfrak{s p}_{2 n-2}$ \\
\hline
\end{tabular}

TABLE IV. The embeddings $\mathfrak{h} \subseteq \mathfrak{g}$ are obtained from [21, Table 1] and [7, Theorem $0]$, which describe each embedding on the level of algebraic groups.

Together with Proposition 35, this result classifies the a-regular, strictly indecomposable reductive spherical pairs. A natural next step is to study the indecomposable reductive spherical pairs that are $\mathfrak{a}$-regular, for which we need the following lemma.

Lemma 39. Let $(\mathfrak{g}, \mathfrak{h})$ be a strictly indecomposable reductive spherical pair. If $(\mathfrak{g},[\mathfrak{h}, \mathfrak{h}])$ is $\mathfrak{a}$-regular, then $(\mathfrak{g}, \mathfrak{h})$ is $\mathfrak{a}$-regular.

Proof. The statement is obviously true if $\mathfrak{h}$ is semisimple, so we assume $\mathfrak{h}$ to be non-semisimple. Let us prove the statement by contraposition, assuming that $(\mathfrak{g}, \mathfrak{h})$ is a strictly indecomposable reductive 
spherical pair that is not $\mathfrak{a}$-regular. At the same time, $\mathfrak{h}$ being non-semisimple and an inspection of [34, Table 26.3], [21, Table 1], and [3, Table 1] reveal that $(\mathfrak{g}, \mathfrak{h})$ is conjugate to one of the pairs in Table $\mathrm{V}$ below. It therefore suffices to prove the following claim: if $(\mathfrak{g}, \mathfrak{h})$ is conjugate to a pair in Table \, then $(\mathfrak{g},[\mathfrak{h}, \mathfrak{h}])$ is not $\mathfrak{a}$-regular.

\begin{tabular}{c|c|c} 
& $\mathfrak{g}$ & $\mathfrak{h}$ \\
\hline 1 & $\mathfrak{s l}_{p+q}(|p-q|>1)$ & $\mathfrak{s l}_{p} \oplus \mathfrak{s l}_{q} \oplus \mathbb{C}$ \\
\hline 2 & $\mathfrak{s o}_{2 n}$ & $\mathfrak{g l}_{n}$ \\
\hline 3 & $\mathfrak{e}_{6}$ & $\mathfrak{s o}(10) \oplus \mathbb{C}$ \\
\hline 4 & $\mathfrak{e}_{7}$ & $\mathfrak{e}_{6} \oplus \mathbb{C}$ \\
\hline 5 & $\mathfrak{s p}_{2 n}(n>2)$ & $\mathfrak{s p}_{2 n-2} \oplus \mathbb{C}$ \\
\hline 6 & $\mathfrak{s o}_{10}$ & $\mathfrak{s o}_{7} \oplus \mathfrak{s 0 _ { 2 }}$ \\
\hline 7 & $\mathfrak{s l}_{n} \oplus \mathfrak{s p}_{2 m} \quad(n>6$ or $m>2)$ & $\mathfrak{g l}_{n-2} \oplus \mathfrak{s l}_{2} \oplus \mathfrak{s p}_{2 m-2}$
\end{tabular}

TABLE V. The embeddings $\mathfrak{h} \subseteq \mathfrak{g}$ are as described in [21, Table 1] and [7, Theorem $0]$, where they are given as embeddings of the corresponding algebraic groups.

Suppose that $(\mathfrak{g}, \mathfrak{h})$ is conjugate to a pair in lines $1,2,3$, or 7 of Table V] It then follows that $(\mathfrak{g},[\mathfrak{h}, \mathfrak{h}])$ is a strictly indecomposable reductive spherical pair, as it appears in at least one of the classifications of Krämer [21], Mikityuk [28, and Brion [7]. At the same time, one can verify that $(\mathfrak{g},[\mathfrak{h}, \mathfrak{h}])$ is not conjugate to a pair in Table III or Table IV. Proposition 35 and Lemma 38 then imply that $(\mathfrak{g},[\mathfrak{h}, \mathfrak{h}])$ is not $\mathfrak{a}$-regular.

Now assume that $(\mathfrak{g}, \mathfrak{h})$ is conjugate to one of the remaining pairs in Table $\mathbf{V}$. Let $(G, H)$ be a corresponding reductive spherical pair of groups, and let us take $G$ to be simply-connected. We note that [34, Table 10.2] then provides explicit generators of $\Lambda_{+}(G,[H, H])$. It is now straightforward to apply Lemma 27 and conclude that $(\mathfrak{g},[\mathfrak{h}, \mathfrak{h}])$ is not $\mathfrak{a}$-regular.

We now study the $\mathfrak{a}$-regular, indecomposable reductive spherical pairs. Let $(\mathfrak{g}, \mathfrak{h})$ be an indecomposable reductive spherical pair and note that $(\mathfrak{g},[\mathfrak{h}, \mathfrak{h}])$ has the following form (cf. Remark [22):

$$
(\mathfrak{g},[\mathfrak{h}, \mathfrak{h}])=\left(\bigoplus_{i=1}^{n} \mathfrak{g}_{i}, \bigoplus_{i=1}^{n} \widetilde{\mathfrak{h}}_{i}\right),
$$

where for all $i \in\{1, \ldots, n\}, \widetilde{\mathfrak{h}}_{i}$ is a semisimple ideal in $[\mathfrak{h}, \mathfrak{h}], \mathfrak{g}_{i}$ is a reductive ideal in $\mathfrak{g}$ containing $\widetilde{\mathfrak{h}}_{i}$, and $\left(\mathfrak{g}_{i}, \widetilde{\mathfrak{h}}_{i}\right)$ is indecomposable. Note that each pair $\left(\mathfrak{g}_{i}, \widetilde{\mathfrak{h}}_{i}\right)$ is actually strictly indecomposable, owing to the fact that $\widetilde{\mathfrak{h}}_{i}$ is semisimple.

Let $\pi_{i}: \mathfrak{g} \rightarrow \mathfrak{g}_{i}$ denote the projection onto the $i^{\text {th }}$ factor and set $\mathfrak{z}_{i}:=\pi_{i}(\mathfrak{z}(\mathfrak{h}))$, where $\mathfrak{z}(\mathfrak{h})$ is the center of $\mathfrak{h}$. It is clear that $\mathfrak{z}_{i}$ is reductive and that it commutes with $\widetilde{\mathfrak{h}}_{i}$, from which we conclude that $\overline{\mathfrak{h}}_{i}:=\widetilde{\mathfrak{h}}_{i} \oplus \mathfrak{z} i \subseteq \mathfrak{g}_{i}$ is a reductive subalgebra. Now set

$$
\overline{\mathfrak{h}}:=\bigoplus_{i=1}^{n} \overline{\mathfrak{h}}_{i} \subseteq \mathfrak{g} .
$$

It follows by construction that $[\mathfrak{h}, \mathfrak{h}] \subseteq \overline{\mathfrak{h}}$ and $\mathfrak{z}(\mathfrak{h}) \subseteq \bigoplus_{i=1}^{n} \mathfrak{z} i \subseteq \overline{\mathfrak{h}}$, implying that $\mathfrak{h} \subseteq \overline{\mathfrak{h}}$ and $\widetilde{\mathfrak{b}}+\mathfrak{h} \subseteq \widetilde{\mathfrak{b}}+\overline{\mathfrak{h}}$ for any Borel subalgebra $\widetilde{\mathfrak{b}} \subseteq \mathfrak{g}$. Since $(\mathfrak{g}, \mathfrak{h})$ is a reductive spherical pair, the previous sentence shows $(\mathfrak{g}, \overline{\mathfrak{h}})$ to be a reductive spherical pair. Our next result establishes that $\left(\mathfrak{g}_{i}, \overline{\mathfrak{h}}_{i}\right)$ is a reductive spherical pair for all $i \in\{1, \ldots, n\}$. 
Lemma 40. Let $(\mathfrak{g}, \mathfrak{h})$ be an indecomposable reductive spherical pair and use the notation from above. Then $\left(\mathfrak{g}_{i}, \overline{\mathfrak{h}}_{i}\right)$ is a strictly indecomposable reductive spherical pair for all $i \in\{1, \ldots, n\}$.

Proof. Since $(\mathfrak{g}, \mathfrak{h})$ is spherical, there exists a Borel subalgebra $\widetilde{\mathfrak{b}} \subseteq \mathfrak{g}$ satisfying $\widetilde{\mathfrak{b}}+\mathfrak{h}=\mathfrak{g}$. The decomposition $\mathfrak{g}=\mathfrak{g}_{1} \oplus \cdots \oplus \mathfrak{g}_{n}$ gives rise to a decomposition of the form $\widetilde{\mathfrak{b}}=\mathfrak{b}_{1} \oplus \cdots \oplus \mathfrak{b}_{n}$, where $\mathfrak{b}_{i}$ is a Borel subalgebra of $\mathfrak{g}_{i}$ for all $i \in\{1, \ldots, n\}$. Now note that $\mathfrak{b}_{i}+\overline{\mathfrak{h}}_{i}=\mathfrak{g}_{i}$ for all $i \in\{1, \ldots, n\}$ if and only if $\widetilde{\mathfrak{b}}+\overline{\mathfrak{h}}=\mathfrak{g}$. Recalling that $(\mathfrak{g}, \overline{\mathfrak{h}})$ is a reductive spherical pair, the previous sentence implies that $\left(\mathfrak{g}_{i}, \overline{\mathfrak{h}}_{i}\right)$ is a reductive spherical pair for all $i \in\{1, \ldots, n\}$.

To complete the proof, we observe that $\left[\overline{\mathfrak{h}}_{i}, \overline{\mathfrak{h}}_{i}\right]=\left\{\widetilde{\mathfrak{h}}_{i}\right.$ for all $i \in\{1, \ldots, n\}$. The strict indecomposability of $\left(\mathfrak{g}_{i}, \overline{\mathfrak{h}}_{i}\right)$ thus follows from the indecomposability of $\left(\mathfrak{g}, \widetilde{\mathfrak{h}}_{i}\right)$.

We may now relate the $\mathfrak{a}$-regularity of $(\mathfrak{g}, \mathfrak{h})$ to that of $(\mathfrak{g}, \overline{\mathfrak{h}})$.

Proposition 41. Let $(\mathfrak{g}, \mathfrak{h})$ be an indecomposable reductive spherical pair and use the notation from above. Then $(\mathfrak{g}, \mathfrak{h})$ is $\mathfrak{a}$-regular if and only if $(\mathfrak{g}, \overline{\mathfrak{h}})$ is $\mathfrak{a}$-regular.

Proof. The inclusion of subalgebras $[\mathfrak{h}, \mathfrak{h}] \subseteq \mathfrak{h} \subseteq \overline{\mathfrak{h}}$ implies the inclusion of Cartan spaces $\mathfrak{a}(\mathfrak{g}, \overline{\mathfrak{h}}) \subseteq$ $\mathfrak{a}(\mathfrak{g}, \mathfrak{h}) \subseteq \mathfrak{a}(\mathfrak{g},[\mathfrak{h}, \mathfrak{h}])$, from which we deduce the backward implication.

For the forward implication, suppose that $(\mathfrak{g}, \mathfrak{h})$ is $\mathfrak{a}$-regular. The inclusion $\mathfrak{a}(\mathfrak{g}, \mathfrak{h}) \subseteq \mathfrak{a}(\mathfrak{g},[\mathfrak{h}, \mathfrak{h}])$ then shows $(\mathfrak{g},[\mathfrak{h}, \mathfrak{h}])$ to be $\mathfrak{a}$-regular, which is equivalent to all of the strictly indecomposable pairs $\left(\mathfrak{g}_{i}, \widetilde{\mathfrak{h}}_{i}\right)$ being $\mathfrak{a}$-regular (see Lemma 21). Since $\left(\mathfrak{g}_{i}, \overline{\mathfrak{h}}_{i}\right)$ is a strictly indecomposable reductive spherical pair (see Lemma 40) with $\left[\overline{\mathfrak{h}}_{i}, \overline{\mathfrak{h}}_{i}\right]=\widetilde{\mathfrak{h}}_{i}$, Lemma 39 implies that $\left(\mathfrak{g}_{i}, \overline{\mathfrak{h}}_{i}\right)$ must be $\mathfrak{a}$-regular. It then follows from Lemma 21 that $(\mathfrak{g}, \overline{\mathfrak{h}})$ is $\mathfrak{a}$-regular.

We now connect this discussion of a-regularity for indecomposable reductive spherical pairs to the overarching objective - a classification of a-regular reductive spherical pairs. The following lemma is a crucial step in this direction.

Lemma 42. If $\mathfrak{h}$ is any reductive subalgebra of $\mathfrak{g}$, then $(\mathfrak{g}, \mathfrak{h})$ is a reductive spherical pair if and only if there exist indecomposable reductive spherical pairs $\left(\mathfrak{g}_{i}, \mathfrak{h}_{i}\right), i \in\{1, \ldots, n\}$, such that $\mathfrak{g}_{i}$ (resp. $\mathfrak{h}_{i}$ ) is an ideal in $\mathfrak{g}$ (resp. $\mathfrak{h})$ for all $i$ and

$$
(\mathfrak{g}, \mathfrak{h})=\left(\bigoplus_{i=1}^{n} \mathfrak{g}_{i}, \bigoplus_{i=1}^{n} \mathfrak{h}_{i}\right) .
$$

Proof. By virtue of Remark 22, one can find indecomposable pairs $\left(\mathfrak{g}_{i}, \mathfrak{h}_{i}\right)$ satisfying the aboveadvertised properties. The proof then becomes entirely analogous to that of Lemma 40.

The classification of a-regular reductive spherical pairs is now described as follows. By virtue of Lemmas 21 and 42, it suffices to classify the indecomposable reductive spherical pairs that are $\mathfrak{a}$-regular. We thus suppose that $(\mathfrak{g}, \mathfrak{h})$ is any indecomposable reductive spherical pair. If $(\mathfrak{g}, \mathfrak{h})$ is strictly indecomposable, then it is a-regular if and only if it is conjugate to a pair in Table [II] or Table IV] If $(\mathfrak{g}, \mathfrak{h})$ is not strictly indecomposable, then we consider the associated pair $(\mathfrak{g}, \overline{\mathfrak{h}})$. The $\mathfrak{a}$-regularity of $(\mathfrak{g}, \mathfrak{h})$ is then equivalent to that of $(\mathfrak{g}, \overline{\mathfrak{h}})$ (see Proposition 41). This is in turn equivalent to every strictly indecomposable pair $\left(\mathfrak{g}_{i}, \overline{\mathfrak{h}}_{i}\right)$ being $\mathfrak{a}$-regular (see Lemma 21), which can be assessed via Tables III and IV.

Remark 43. One might ask about the feasibility of classifying the a-regular reductive spherical pairs $(G, H)$ satisfying $c_{G}(G / H)>0$. The complexity-one case might be tractable, largely because the papers 22 and [31] classify all strictly indecomposable reductive spherical pairs $(G, H)$ with $c_{G}(G / H)=1$. One can thereby determine which of the strictly indecomposable, complexity-one pairs are a-regular. In analogy with 5.5.2 and 5.5.3, this might imply a classification of all reductive spherical $(G, H)$ with $c_{G}(G / H)=1$. The case of $c_{G}(G / H)>1$ remains unclear to us. 


\section{REFERENCES}

[1] Abe, H., And CRooks, P. Hessenberg varieties, Slodowy slices, and integrable systems. arxiv:1807.07792 (2018), 36pp. To appear in Math. Z.

[2] Arzhantsev, I. V., And Chuvashova, O. V. Classification of affine homogeneous spaces of complexity one. Mat. Sb. 195, 6 (2004), 3-20.

[3] Avdeev, R. S. Extended weight semigroups of affine spherical homogeneous spaces of nonsimple semisimple algebraic groups. Izv. Ross. Akad. Nauk Ser. Mat. 74, 6 (2010), 3-26.

[4] Bielawski, R. Hyperkähler structures and group actions. J. London Math. Soc. (2) 55, 2 (1997), 400-414.

[5] Bielawski, R. Slices to sums of adjoint orbits, the Atiyah-Hitchin manifold, and Hilbert schemes of points. Complex Manifolds 4 (2017), 16-36.

[6] Biquard, O. Sur les équations de Nähm et la structure de Poisson des algèbres de Lie semi-simples complexes. Math. Ann. 304, 2 (1996), 253-276.

[7] Brion, M. Classification des espaces homogènes sphériques. Compositio Math. 63, 2 (1987), 189-208.

[8] Crooks, P. An equivariant description of certain holomorphic symplectic varieties. Bull. Aust. Math. Soc. 97, 2 (2018), 207-214.

[9] Crooks, P., AND RAYAn, S. Abstract integrable systems on hyperkähler manifolds arising from Slodowy slices. arxiv:1706.05819 (2017), 17pp. To appear in Math. Res. Lett.

[10] Dancer, A., And Swann, A. Hyperkähler metrics associated to compact Lie groups. Math. Proc. Cambridge Philos. Soc. 120, 1 (1996), 61-69.

[11] Dynkin, E. B. Semisimple subalgebras of semisimple Lie algebras. Transl., Ser. 2, Am. Math. Soc. 6 (1957), $111-243$.

[12] Èlašvili, A. G. Canonical form and stationary subalgebras of points in general position for simple linear Lie groups. Funkcional. Anal. i Priložen. 6, 1 (1972), 51-62.

[13] Èlašvili, A. G. Stationary subalgebras of points of general position for irreducible linear Lie groups. Funkcional. Anal. i Priložen. 6, 2 (1972), 65-78.

[14] Hitchin, N. J. The self-duality equations on a Riemann surface. Proc. London Math. Soc. (3) 55, 1 (1987), 59-126.

[15] Hitchin, N. J., Karlhede, A., Lindström, U., And RočEK, M. Hyperkähler metrics and supersymmetry. Comm. Math. Phys. 108, 4 (1987), 535-589.

[16] Knop, F. Weylgruppe und Momentabbildung. Invent. Math. 99, 1 (1990), 1-23.

[17] Knop, F. The asymptotic behavior of invariant collective motion. Invent. Math. 116, 1-3 (1994), $309-328$.

[18] Kostant, B. Lie group representations on polynomial rings. Amer. J. Math. 85 (1963), 327-404.

[19] Kovalev, A. G. Nähm's equations and complex adjoint orbits. Quart. J. Math. Oxford Ser. (2) 47, 185 (1996), $41-58$.

[20] Krämer, M. Eine Klassifikation bestimmter Untergruppen kompakter zusammenhängender Liegruppen. Comm. Algebra 3, 8 (1975), 691-737.

[21] Krämer, M. Sphärische Untergruppen in kompakten zusammenhängenden Liegruppen. Compositio Math. 38, 2 (1979), 129-153.

[22] Kronheimer, P. A hyperkähler structure on the cotangent bundle of a complex Lie group. arXiv:math/0409253 (2004), $11 \mathrm{pp}$.

[23] Kronheimer, P. B. A hyperkählerian structure on coadjoint orbits of a semisimple complex group. J. London Math. Soc. (2) 42, 2 (1990), 193-208.

[24] Kronheimer, P. B. Instantons and the geometry of the nilpotent variety. J. Differential Geom. 32, 2 (1990), $473-490$

[25] Losev, I. V. Computation of Cartan spaces for affine homogeneous spaces. Mat. Sb. 198, 10 (2007), 31-56.

[26] Marsden, J. E., Ratiu, T., and Raugel, G. Symplectic connections and the linearisation of Hamiltonian systems. Proc. Roy. Soc. Edinburgh Sect. A 117, 3-4 (1991), 329-380.

[27] Maynand, M. Stratified hyperkähler spaces from semisimple Lie algebras. arXiv:1709.09126 (2017), 19 pp. To appear in Transformation Groups.

[28] Mikityuk, I. V. Integrability of invariant Hamiltonian systems with homogeneous configuration spaces. Mat. Sb. (N.S.) 129(171), 4 (1986), 514-534, 591.

[29] Nakajima, H. Instantons on ALE spaces, quiver varieties, and Kac-Moody algebras. Duke Math. J. 76, 2 (1994), 365-416.

[30] Nakajima, H. Quiver varieties and Kac-Moody algebras. Duke Math. J. 91, 3 (1998), 515-560.

[31] Panyushev, D. I. Complexity and rank of actions in invariant theory. J. Math. Sci. (New York) 95, 1 (1999), 1925-1985. Algebraic geometry, 8.

[32] Richardson, JR., R. W. Principal orbit types for algebraic transformation spaces in characteristic zero. Invent. Math. 16 (1972), 6-14. 
[33] Tauvel, P., And Yu, R. W. T. Lie algebras and algebraic groups. Springer Monographs in Mathematics. SpringerVerlag, Berlin, 2005.

[34] Timashev, D. A. Homogeneous spaces and equivariant embeddings, vol. 138 of Encyclopaedia of Mathematical Sciences. Springer, Heidelberg, 2011. Invariant Theory and Algebraic Transformation Groups, 8.

(Crooks) Department of Mathematics, Northeastern University, 360 Huntington Ave., Boston, MA 02115, USA

E-mail address: peter.d.crooks@gmail.com

(van Pruijssen) Institut für Mathematik, University of Paderborn, Warburger Str. 100, 33098 PaderBORn, GERMAnY

E-mail address: vanpruijssen@math.upb.de 